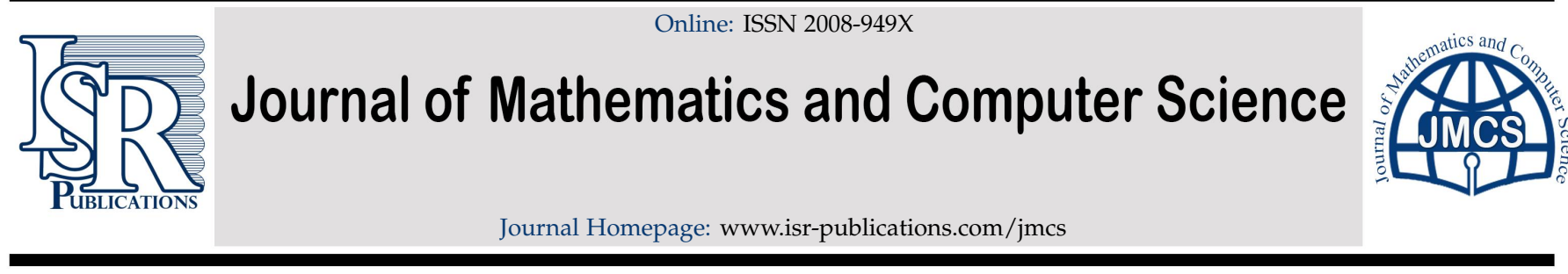

\title{
On fuzzy soft b-open sets in fuzzy soft bitopological space
}

\author{
A. F. Sayed \\ Mathematics Department, Al-Lith University College, Umm Al-Qura University, P. O. Box 112, Al-Lith 21961, Makkah Al Mukarramah, \\ Kingdom of Saudi Arabia.
}

\begin{abstract}
In this paper, we introduce $(1,2)^{*}$-fuzzy soft b-open sets and $(1,2)^{*}$-fuzzy soft b-closed sets in fuzzy soft bitopological space and exhibit the properties of $(1,2)^{*}$-fuzzy soft $b$ - open sets. Then we discuss the relation with $(1,2)^{*}$-fuzzy soft regular- open, $(1,2)^{*}$-fuzzy soft pre-open, $(1,2)^{*}$-fuzzy soft semi-open, $(1,2)^{*}$-fuzzy soft $\alpha$-open, and $(1,2)^{*}$-fuzzy soft $\beta$-open in fuzzy soft bitopological space. Also, we introduce and investigate the concepts of $(1,2)^{*}$-fuzzy soft b-interior, $(1,2)^{*}$-fuzzy soft b-closure in fuzzy soft bitopological space and analyze the relations between these concepts.
\end{abstract}

Keywords: $(1,2)^{*}$-fuzzy soft b-open set, $(1,2)^{*}$-fuzzy soft b-closed set, $(1,2)^{*}$-fuzzy soft regular-open, $(1,2)^{*}$-fuzzy soft pre open, $(1,2)^{*}$-fuzzy soft semi open, $(1,2)^{*}$-fuzzy soft $\alpha$ - open, $(1,2)^{*}$-fuzzy soft $\beta$-open, $(1,2)^{*}$-fuzzy soft b-interior, $(1,2)^{*}$-fuzzy soft b-closure.

2020 MSC: 54A05, 54A40, 54F99.

(C)2020 All rights reserved.

\section{Introduction}

In the year 1965, Zadeh [25], introduced the concept of fuzzy set theory and its applications can be found in many branches of mathematical and engineering sciences including management science, control engineering, computer science, artificial intelligence (see, $[5,6])$.

In the year 1999, Russian researcher Molodtsov [14], initiated the concept of soft sets as a new mathematical tool to deal with uncertainties while modeling problems in engineering physics, computer science, economics, social sciences and medical sciences (see, [16, 24]). In 2003, Maji et al. [13], studied the theory of soft sets initiated by Molodtsov. They defined equality of two soft sets, subset and super set of a soft set, complement of a soft set, null soft set and absolute soft set with examples. Soft binary operations like AND, OR and also the operations of union and intersection were also defined. In 2005, Chen [4], presented a new definition of soft set parametrization reduction and a comparison of it with attribute reduction in rough set theory.

In 1963, Kelly [10], first initiated the concept of bitopological spaces and other authors have contributed to development and construction some properties of such spaces (see, [17, 18]) as a generalizations of which are in general topology.

Email address: dr.afsayed@hotmail.com, afssayed@uqu.edu.sa (A. F. Sayed)

doi: 10.22436/jmcs.021.01.04

Received: 2019-10-22 Revised: 2020-02-02 Accepted: 2020-02-10 
In 2014, Ittanagi [7], introduced and studied the concept of soft bitopological spaces and other authors have contributed to development and construction some properties of such spaces (see, $[8,9,19])$. The notion of a soft bitopological space was introduced using different soft topologies on an initial universe set. On the other hand, the mixed type of soft set theory was given using different soft topologies (see, $[1,2,23])$.

In 2015, Mukherjee1 and Park [15] were first introduced the notion of fuzzy soft bitopological space and they introduced the notions of $\tau_{1} \tau_{2}$-fuzzy soft open(closed) sets, $\tau_{1} \tau_{2}$-fuzzy soft interior (resp. closure) and studied some of their basic properties. Also, my work in $([21,22])$ was extension and continuation of studying in this trend by characterizing a new type of fuzzy soft sets and introducing some separation axioms in fuzzy soft bitopological spaces. In this paper, we introduce $(1,2)^{*}$-fuzzy soft b-open sets and $(1,2)^{*}$-fuzzy soft b-closed sets in fuzzy soft bitopological space and exhibit the properties of $(1,2)^{*}$-fuzzy soft $b$ - open sets. Then we discuss the relation with $(1,2)^{*}$-fuzzy soft regular- open, $(1,2)^{*}$-fuzzy soft pre-open, $(1,2)^{*}$-fuzzy soft semi open, $(1,2)^{*}$-fuzzy soft $\alpha$-open, $(1,2)^{*}$-fuzzy soft $\beta$-open in fuzzy soft bitopological space. Also we introduced and investigated the concepts of $(1,2)^{*}$-fuzzy soft b-interior, $(1,2)^{*}$-fuzzy soft b-closure in fuzzy soft bitopological space and analyze the relations between these concepts.

\section{Preliminaries}

Definition 2.1 ([25]). A fuzzy set $A$ in a non-empty set $X$ is characterized by a membership function $\mu_{A}: X \rightarrow[0,1]=$ I whose value $\mu_{A}(x)$ represents the "degree of membership" of $x$ in $A$ for every $x$ in $X$. Let $\mathrm{I}^{\mathrm{X}}$ denotes the family of all fuzzy sets on $\mathrm{X}$.

A member $A$ in $I^{X}$ is contained in a member $B$ of $I^{X}$ denoted $A \leqslant B$ if and only if $\mu_{A}(x) \leqslant \mu_{B}(x)$ for every $x \in X$ (see [25]).

Let $A, B \in I^{X}$, we have the following fuzzy sets (see [25]).

(1) Equality: $A=B$ if and only if $\mu_{A}(x)=\mu_{B}(x)$ for all $x \in X$.

(2) Intersection: $C=A \wedge B \in I^{X}$ by $\mu_{C}(x)=\min \left\{\mu_{A}(x), \mu_{B}(x)\right\}$ for all $x \in X$.

(3) Union: $\mathrm{D}=\mathrm{A} \vee \mathrm{B} \in \mathrm{I}^{\mathrm{X}}$ by $\mu_{\mathrm{C}}(\mathrm{x})=\max \left\{\mu_{\mathrm{A}}(\mathrm{x}), \mu_{\mathrm{B}}(\mathrm{x})\right\}$ for all $\mathrm{x} \in \mathrm{X}$.

(4) Complement: $E=A^{c} \in I^{X}$ by $\mu_{E}(x)=1-\mu_{A}(x)$ for all $x \in X$.

Definition 2.2 ([25]). The empty fuzzy set on $X$ denoted by $\tilde{0}$ is a function which maps each $x \in X$ to 0 . That is, $\tilde{0}(x)=0$ for all $x \in X$. A universal fuzzy set denoted by $\tilde{1}$ is a function which maps each $x \in X$ to 1. That is, $\tilde{1}(x)=1$ for all $x \in X$.

Definition 2.3 ([14]). Let $A \subseteq E$. A pair $(F, A)$ is called a soft set over $X$ if $F$ is a mapping $F: A \rightarrow P(X)$.

Definition 2.4 ([12]). Let $A \subseteq E$. A pair $(f, A)$, denoted by $f_{A}$, is called a fuzzy soft set over $X$, where $f$ is a mapping given by $f: A \rightarrow I^{X}$ defined by $f_{A}(e)=\mu_{f_{A}}^{e}$ where $\mu_{f_{A}}^{e}= \begin{cases}\tilde{0}, & \text { if } e \notin A, \\ \text { otherwise, } & \text { if } e \in A .\end{cases}$

$\widetilde{(X, E)}$ denotes the family of all fuzzy soft sets over $(X, E)$.

Definition 2.5 ([13]). A fuzzy soft set $f_{A} \tilde{\in(X, E)}$ is said to be:

(a) NULL fuzzy soft set, denoted by $\tilde{\phi}$, if for all $e \in A, f_{A}(e)=\tilde{0}$;

(b) absolute fuzzy soft set, denoted by $\tilde{E}$, if for all $e \in E, f_{A}(e)=\tilde{1}$.

Definition 2.6 ([20]). The complement of a fuzzy soft set $f_{A}$, denoted by $f_{A}^{c}$ where $f_{A}^{c}: E \rightarrow I^{X}$ is a mapping given by $\mu_{f_{A}^{c}}^{e}=\tilde{1}-\mu_{f_{A}}^{e}$, for all $e \in E$ and where $\tilde{1}(x)=1$, for all $x \in X$. Clearly $\left(f_{A}^{c}\right)^{c}=f_{A}$.

Definition 2.7 ([20]). Let $f_{A}, g_{B} \in \widetilde{(X, E)}$. $f_{A}$ is fuzzy soft subset of $g_{B}$, denoted by $f_{A} \tilde{\subseteq} g_{B}$, if $A \subseteq B$ and $\mu_{f_{A}}^{e} \leqslant \mu_{g_{B}}^{e}$ for all $e \in A$, i.e., $\mu_{f_{A}}^{e}(x) \leqslant \mu_{g_{B}}^{e}(x)$ for all $x \in X$ and for all $e \in A$. 
Definition 2.8 ([20]). Let $f_{A}, g_{B} \tilde{\in} \widetilde{(X, E)}$. The union of $f_{A}$ and $g_{B}$ is also a fuzzy soft set $h_{C}$, where $C=A \cup B$ and for all $e \in C, h_{C}(e)=\mu_{h_{c}}^{e}=\mu_{f_{A}}^{e} \vee \mu_{g_{B}}^{e}$. Here we write $h_{C}=f_{A} \tilde{\cup} g_{B}$.

Definition 2.9. [20] Let $f_{A}, g_{B} \tilde{\in} \widetilde{(X, E)}$. The intersection of $f_{A}$ and $g_{B}$ is also a fuzzy soft set $d_{C}$, where $C=A \cap B$ and for all $e \in C, d_{C}(e)=\mu_{d_{c}}^{e}=\mu_{f_{A}}^{e} \wedge \mu_{g_{B}}^{e}$. Here we write $d_{C}=f_{A} \tilde{\cap} g_{B}$.

Definition 2.10 ([11]). The fuzzy soft set $f_{A} \tilde{\in} \widetilde{(X, E)}$ is called fuzzy soft point if there exist $x \in X$ and $e \in E$ such that $\mu_{f_{A}}^{e}(x)=\alpha(0<\alpha \leqslant 1)$ and $\mu_{f_{A}}^{e}(y)=0$ for each $y \in X-\{x\}$, and this fuzzy soft point is denoted by $x_{\alpha}^{e}$ or $f_{e}$.

Definition 2.11 ([11]). The fuzzy soft point $f_{e}$ is said to be belonging to the fuzzy soft set $(g, A)$, denoted by $f_{e} \tilde{\in}(g, A)$, if for the element $e \in A, \alpha \leqslant \mu_{g_{A}}^{e}(x),(0<\alpha \leqslant 1)$.

Definition 2.12 ([3]). Let $f_{A}$ be fuzzy soft set over $X$. The two fuzzy soft points $f_{e_{1}}, f_{e_{2}} \tilde{\in} f_{A}$ are said to be equal if $\mu_{\mathrm{f}_{e_{1}}}(x)=\mu_{\mathrm{f}_{e_{2}}}(x)$ for all $x \in X$. Thus $f_{e_{1}} \neq f_{e_{2}}$ if and only $\mu_{f_{e_{1}}}(x) \neq \mu_{f_{e_{2}}}(x)$ for all $x \in X$.

Definition 2.13 ([20]). A fuzzy soft topology $\tau$ over $(X, E)$ is a family of fuzzy soft sets over $(X, E)$ satisfying the following properties

(i) $\tilde{0}_{\mathrm{E}}, \tilde{1}_{\mathrm{E}} \in \tau$;

(ii) if $f_{A}, g_{B} \in \tau$, then $f_{A} \tilde{\cap} g_{B} \in \tau$;

(iii) if $f_{A_{\alpha}} \in \tau$ for all $\alpha \in \Delta$ an index set, then $\tilde{U}_{\alpha \in \Delta} f_{A_{\alpha}} \in \tau$.

Definition 2.14 ([15]). If $\tau$ is a fuzzy soft topology on $(X, E)$; the triple $(X, E, \tau)$ is said to be a fuzzy soft topological space. Also each member of $\tau$ is called a fuzzy soft open set in $(X, E, \tau)$.

The complement of a fuzzy soft open set is a fuzzy soft closed set.

Definition 2.15 ([15]). Let $\left(X, E, \tau_{1}\right)$ and $\left(X, E, \tau_{2}\right)$ be two different fuzzy soft topologies on $(X, E)$. Then $\left(X, E, \tau_{1}, \tau_{2}\right)$ is called a fuzzy soft bitopological space on which no separation axioms are assumed unless explicitly stated.

The members of $\tau_{i}(i=1,2)$ are called $\tau_{i}(i=1,2)$-fuzzy soft open sets and the complement of $\tau_{i}(i=$ $1,2)$-fuzzy soft open sets are called $\tau_{i}(i=1,2)$-fuzzy soft closed sets.

Definition 2.16 ([15]). A fuzzy soft set $f_{E} \tilde{\in(X, E)}$ is called $\tau_{1} \tau_{2}$-fuzzy soft open set if $f_{E}=g_{E} \tilde{U} h_{E}$ such that $g_{E} \tilde{\in} \tau_{1}$ and $h_{E} \tilde{\in} \tau_{2}$.

The complement of $\tau_{1} \tau_{2}$-fuzzy soft open set is called $\tau_{1} \tau_{2}$-fuzzy soft closed set.

The family of all $\tau_{1} \tau_{2}$-fuzzy soft open (closed) sets in $\left(X, E, \tau_{1}, \tau_{2}\right)$ is denoted by $\tau_{1} \tau_{2} \operatorname{FSO}\left(X, \tau_{1}, \tau_{2}\right)_{E}$ $\left(\tau_{1} \tau_{2} \mathrm{FSC}\left(\mathrm{X}, \tau_{1}, \tau_{2}\right)_{\mathrm{E}}\right)$, respectively.

Remark 2.17 ([15]). $\tau_{\mathfrak{i}}(i=1,2)$-fuzzy soft open is $\tau_{1} \tau_{2}$-fuzzy soft open but the converse is not true.

Theorem 2.18 ([15]). If $\left.\left(X, E, \tau_{1}, \tau_{2}\right)\right)$ is a fuzzy soft bitopological space, then $\tau=\tau_{1} \tilde{\cap} \tau_{2}$ is a fuzzy soft topological space over $(\mathrm{X}, \mathrm{E})$.

Remark 2.19 ([15]). If $\left(X, E, \tau_{1}, \tau_{2}\right)$ is a fuzzy soft bitopological space, then $\tau=\tau_{1} \tilde{\cup} \tau_{2}$ is not a fuzzy soft topological space over $(X, E)$.

Definition 2.20 ([15]). Let $\left(X, E, \tau_{1}, \tau_{2}\right)$ be a fuzzy soft bitopological space and $f_{E} \widetilde{\in} \widetilde{(X, E)}$. Then the $\tau_{i}(i=$ 1,2)-fuzzy soft closure of $f_{E}$, denoted by $\tau_{i} c l\left(f_{E}\right)$, is the intersection of all $\tau_{i}(i=1,2)$-fuzzy soft closed supersets of $f_{E}$.

Clearly, $\tau_{i} \operatorname{cl}\left(f_{E}\right)$ is the smallest $\tau_{i}(i=1,2)$ - fuzzy soft closed set over $(X, E)$ which contains $f_{E}$. 
Definition 2.21 ([15]). Let $\left(X, E, \tau_{1}, \tau_{2}\right)$ be a fuzzy soft bitopological space and $f_{E} \widetilde{\in(X, E)}$. Then the $\tau_{1} \tau_{2}-$ fuzzy soft closure of $f_{E}$, denoted by $\tau_{1} \tau_{2} c l\left(f_{E}\right)$, is the intersection of all $\tau_{1} \tau_{2}$-fuzzy soft closed supersets of $f_{E}$.

Clearly, $\tau_{1} \tau_{2} \operatorname{cl}\left(f_{E}\right)$ is the smallest $\tau_{1} \tau_{2}$-fuzzy soft closed set over $(X, E)$ which contains $f_{E}$.

Remark 2.22 ([15]). If $\left(X, E, \tau_{1}, \tau_{2}\right)$ is a fuzzy soft bitopological space and $f_{E} \widetilde{E}(X, E)$, then $\tau_{1} \tau_{2} \operatorname{cl}\left(f_{E}\right) \tilde{\tilde{E}} \tau_{i} \operatorname{cl}\left(f_{E}\right)$ $(i=1,2)$.

Definition $2.23([15])$. Let $\left(X, E, \tau_{1}, \tau_{2}\right)$ be a fuzzy soft bitopological space and $f_{E} \widetilde{\in} \widetilde{(X, E)}$. Then the $\tau_{i}(i=$ $1,2)$-fuzzy soft interior of $f_{E}$, denoted by $\tau_{i} \operatorname{int}\left(f_{E}\right)$, is the union of all $\tau_{i}(i=1,2)$-fuzzy soft open subsets of $f_{E}$.

Clearly, $\tau_{i} \operatorname{int}\left(f_{E}\right)$ is the largest $\tau_{i}(i=1,2)$ - fuzzy soft open set over $(X, E)$ which contained in $f_{E}$.

Definition $2.24([15])$. Let $\left(X, E, \tau_{1}, \tau_{2}\right)$ be a fuzzy soft bitopological space and $f_{E} \widetilde{\in} \widetilde{(X, E)}$. Then the $\tau_{1} \tau_{2^{-}}$ fuzzy soft interior of $f_{E}$, denoted by $\tau_{1} \tau_{2} \operatorname{int}\left(f_{E}\right)$, is the union of all $\tau_{1} \tau_{2}$-fuzzy soft open subsets of $f_{E}$.

Clearly, $\tau_{1} \tau_{2} \operatorname{cl}\left(f_{E}\right)$ is the largest $\tau_{1} \tau_{2}$-fuzzy soft open set over $(X, E)$ which contained in $f_{E}$.

Remark 2.25 ([15]). If $\left(X, E, \tau_{1}, \tau_{2}\right)$ is a fuzzy soft bitopological space and $f_{E} \tilde{\in} \widetilde{(X, E)}$, then $\tau_{i} \operatorname{int}\left(f_{E}\right)(i=$ $1,2) \tilde{\widetilde{\subseteq}} \tau_{1} \tau_{2} \operatorname{int}\left(f_{E}\right)$.

Definition 2.26 ([21]). Let $\left(X, E, \tau_{1}, \tau_{2}\right)$ be a soft bitopological space. Then, the family of all $\tau_{1} \tau_{2}$-fuzzy soft open sets is a supra fuzzy soft topology on $(X, E)$. This supra fuzzy soft topology, will denoted by $\tau_{12}$, i.e., $\tau_{12}=\tau_{1} \tau_{2} \operatorname{FSO}\left(X, \tau_{1}, \tau_{2}\right)_{E}=\left\{g_{E}=g_{1_{E}} \tilde{\sim} g_{2_{E}}: g_{i_{E}} \tilde{E} \tau_{i}, i=1,2\right\}$ and the triple $\left(X, E, \tau_{12}\right)$ is the supra fuzzy soft topological space associated to the fuzzy soft bitopological space $\left(X, E, \tau_{1}, \tau_{2}\right)$.

\section{3. $(1,2)^{*}$-fuzzy soft b-open sets}

In this section we introduce $(1,2)^{*}$-fuzzy soft b-open sets in fuzzy soft bitopological spaces and study some of their properties.

We begin by the following concepts which will be used in the sequal.

Definition 3.1. A fuzzy soft set $f_{E}$ in a fuzzy soft bitopological space $\left(X, E, \tau_{1}, \tau_{2}\right)$ is called

(i) $(1,2)^{*}$-fuzzy soft regular open set if $f_{E}=\tau_{1} \tau_{2}$ int $\left(\tau_{1} \tau_{2} c l\left(f_{E}\right)\right)$ and $(1,2)^{*}$-fuzzy soft regular closed set if $f_{E}=\tau_{1} \tau_{2} c l\left(\tau_{1} \tau_{2} \operatorname{int}\left(f_{E}\right)\right)$;

(ii) $(1,2)^{*}$-fuzzy soft $\alpha$-open set if $f_{E} \tilde{\subseteq} \tau_{1} \tau_{2} \operatorname{int}\left(\tau_{1} \tau_{2} c l\left(\tau_{1} \tau_{2} \operatorname{int}\left(f_{E}\right)\right)\right)$ and $(1,2)^{*}$-fuzzy soft $\alpha$-closed set if $\tau_{1} \tau_{2} \operatorname{cl}\left(\tau_{1} \tau_{2} \operatorname{int}\left(\tau_{1} \tau_{2} \operatorname{cl}\left(f_{E}\right)\right)\right) \tilde{\subseteq} f_{E} ;$

(iii) $(1,2)^{*}$-fuzzy soft pre open set if $f_{E} \tilde{\subseteq} \tau_{1} \tau_{2} \operatorname{int}\left(\tau_{1} \tau_{2} c l\left(f_{E}\right)\right)$ and $(1,2)^{*}$-fuzzy soft pre closed set if $\tau_{1} \tau_{2} \operatorname{cl}\left(\tau_{1} \tau_{2} \operatorname{int}\left(f_{E}\right)\right) \tilde{\subseteq} f_{E}$

(iv) $(1,2)^{*}$-fuzzy soft semi open set if $f_{E} \tilde{\subseteq} \tau_{1} \tau_{2} c l\left(\tau_{1} \tau_{2} \operatorname{int}\left(f_{E}\right)\right)$ and $(1,2)^{*}$-fuzzy soft semi closed set if $\tau_{1} \tau_{2} \operatorname{int}\left(\tau_{1} \tau_{2} \operatorname{cl}\left(f_{E}\right)\right) \tilde{\subseteq} f_{E}$

(v) $(1,2)^{*}$-fuzzy soft $\beta$-open set if $f_{E} \tilde{\subseteq} \tau_{1} \tau_{2} c l\left(\tau_{1} \tau_{2} \operatorname{int}\left(\tau_{1} \tau_{2} \mathrm{cl}\left(f_{E}\right)\right)\right)$ and $(1,2)^{*}$-fuzzy soft $\beta$-closed set if $\tau_{1} \tau_{2} \operatorname{int}\left(\tau_{1} \tau_{2} \operatorname{cl}\left(\tau_{1} \tau_{2} \operatorname{int}\left(f_{E}\right)\right)\right) \tilde{\subseteq} f_{E}$.

The family of all $(1,2)^{*}$-fuzzy soft regular open(resp. $(1,2)^{*}$-fuzzy soft $\alpha$-open, $(1,2)^{*}$-fuzzy soft preopen, $(1,2)^{*}$-fuzzy soft semi open, $(1,2)^{*}$-fuzzy soft $\beta$-open) sets will be denoted by $(1,2)^{*}$-fsr open(resp. $(1,2)^{*}$-fs $\alpha$ open, $(1,2)^{*}$-fsp open, $(1,2)^{*}$-fss open, $(1,2)^{*}$-fs $\beta$ open) sets.

Lemma 3.2. Let $\left(\mathrm{X}, \mathrm{E}, \tau_{1}, \tau_{2}\right)$ be a fuzzy soft bitopological space. We have the following results.

(i) Every $(1,2)^{*}$-fuzzy soft regular open set is $(1,2)^{*}$-fuzzy soft open.

(ii) Every $(1,2)^{*}$-fuzzy soft open set is $(1,2)^{*}$-fuzzy soft $\alpha$-open.

(iii) Every $(1,2)^{*}$-fuzzy soft $\alpha$-open set is $(1,2)^{*}$-fuzzy soft semi open. 
(iv) Every $(1,2)^{*}$-fuzzy soft pre open set is $(1,2)^{*}$-fuzzy soft $\beta$-open.

(v) Every $(1,2)^{*}$-fuzzy soft semi open set is $(1,2)^{*}$-fuzzy soft $\beta$-open.

(vi) Every $(1,2)^{*}$-fuzzy soft $\alpha$-open set is $(1,2)^{*}$-fuzzy soft pre open.

Proof.

(i). Let $f_{E}$ be a $(1,2)^{*}$-fuzzy soft regular open set. Then $f_{E}=\tau_{1} \tau_{2} \operatorname{int}\left(\tau_{1} \tau_{2} c l\left(f_{E}\right)\right)$, since $\tau_{1} \tau_{2} c l\left(f_{E}\right)$ is a fuzzy soft closed set in fuzzy soft bitopological space and fuzzy soft interior of any set is fuzzy soft open. Therefore, $f_{E}$ is $(1,2)^{*}$-fuzzy soft open.

(ii). Let $f_{E}$ be a $(1,2)^{*}$-fuzzy soft open set. This implies

$$
f_{E}=\tau_{1} \tau_{2} \operatorname{int}\left(\tau_{1} \tau_{2} c l\left(\tau_{1} \tau_{2} \operatorname{int}\left(f_{E}\right)\right)\right)
$$

since $f_{E} \tilde{\subseteq} \tau_{1} \tau_{2} \operatorname{cl}\left(f_{E}\right)=\tau_{1} \tau_{2} c l\left(\tau_{1} \tau_{2} \operatorname{int}\left(\tau_{1} \tau_{2} c l\left(f_{E}\right)\right)\right)$. Thus, $f_{E}$ is $(1,2)^{*}$-fuzzy soft $\alpha$-open.

(iii). Let $f_{E}$ be a $(1,2)^{*}$-fuzzy soft $\alpha$-open set. This implies

$$
f_{E} \tilde{\subseteq} \tau_{1} \tau_{2} \operatorname{int}\left(\tau_{1} \tau_{2} c l\left(\tau_{1} \tau_{2} \operatorname{int}\left(f_{E}\right)\right)\right) \tilde{\subseteq} \tau_{1} \tau_{2} c l\left(\tau_{1} \tau_{2} \operatorname{int}\left(f_{E}\right)\right) .
$$

Thus, $f_{E}$ is $(1,2)^{*}$-fuzzy soft semi open.

(iv). Let $f_{\mathrm{E}}$ be a $(1,2)^{*}$-fuzzy soft pre-open set. This implies

$$
f_{E} \tilde{\subseteq} \tau_{1} \tau_{2} \operatorname{int}\left(\tau_{1} \tau_{2} \operatorname{cl}\left(f_{E}\right)\right) \tilde{\subseteq} \tau_{1} \tau_{2} \operatorname{cl}\left(\tau_{1} \tau_{2} \operatorname{int}\left(\tau_{1} \tau_{2} \operatorname{cl}\left(f_{E}\right)\right)\right) .
$$

Thus, $f_{E}$ is $(1,2)^{*}$-fuzzy soft $\beta$-open.

(v). Let $f_{E}$ be a $(1,2)^{*}$-fuzzy soft $\alpha$-open set. This implies

$$
f_{E} \tilde{\subseteq} \tau_{1} \tau_{2} \operatorname{cl}\left(\tau_{1} \tau_{2} \operatorname{int}\left(f_{E}\right)\right) \tilde{\subseteq} \tau_{1} \tau_{2} \operatorname{cl}\left(\tau_{1} \tau_{2} \operatorname{cl}\left(f_{E}\right)\right) .
$$

Thus, $f_{E}$ is $(1,2)^{*}$-fuzzy soft $\beta$-open.

(vi). Let $f_{E}$ be a $(1,2)^{*}$-fuzzy soft $\alpha$-open set. This implies

$$
f_{E} \tilde{\subseteq} \tau_{1} \tau_{2} \operatorname{int}\left(\tau_{1} \tau_{2} \operatorname{cl}\left(\tau_{1} \tau_{2} \operatorname{int}\left(f_{E}\right)\right)\right) \tilde{\subseteq} \tau_{1} \tau_{2} \operatorname{int}\left(\tau_{1} \tau_{2} \operatorname{cl}\left(f_{E}\right)\right) .
$$

Thus, $f_{E}$ is $(1,2)^{*}$-fuzzy soft pre-open.

Remark 3.3. The converse of the above lemma is need not be true as shown in the following examples.

Example 3.4. Let $X=\{x, y\}, E=\left\{e_{1}, e_{2}\right\}$ and let $\tau_{1}=\left\{\tilde{0}_{E}, \tilde{1}_{E}, f_{1_{E}}, f_{2_{E}}\right\}, \tau_{2}=\left\{\tilde{0}_{E}, \tilde{1}_{E}, g_{1_{E}}, g_{2_{E}}, g_{3_{E}}\right\}$, where

$$
\begin{aligned}
f_{1_{\mathrm{E}}} & =\left\{f_{1}\left(e_{1}\right)=\{x / 0.0, y / 0.0\}=\tilde{0}, f_{1}\left(e_{2}\right)=\{x / 0.3, y / 0.0\}\right\}, \\
f_{2_{\mathrm{E}}} & =\left\{f_{2}\left(e_{1}\right)=\{x / 0.0, y / 0.7\}, f_{2}\left(e_{2}\right)=\{x / 0.0, y / 0.6\}\right\}, \\
g_{1_{\mathrm{E}}} & =\left\{g_{1}\left(e_{1}\right)=\{x / 0.5, y / 0.0\}, g_{1}\left(e_{2}\right)=\{x / 0.0, y / 0.0\}=\tilde{0}\right\}, \\
g_{2_{\mathrm{E}}} & =\left\{g_{2}\left(e_{1}\right)=\{x / 0.5, y / 0.0\}, g_{2}\left(e_{2}\right)=\{x / 0.3, y / 0.0\}\right\}, \\
g_{3_{\mathrm{E}}} & =\left\{g_{3}\left(e_{1}\right)=\{x / 0.5, y / 0.7\}, g_{3}\left(e_{2}\right)=\{x / 0.3, y / 0.0\}\right\} .
\end{aligned}
$$

It is clear that $\left(\mathrm{X}, \mathrm{E}, \tau_{1}, \tau_{2}\right)$ is a fuzzy soft bitopological space. The $\tau_{1} \tau_{2}-$ fuzzy soft open sets are $\left\{\tilde{0}_{\mathrm{E}}, \tilde{1}_{\mathrm{E}}, \mathrm{f}_{1_{\mathrm{E}}}, \mathrm{f}_{2_{\mathrm{E}}}, \mathrm{g}_{1_{\mathrm{E}}}\right.$, $\left.g_{2_{E}}, g_{3_{E}}, p_{E}, q_{E}\right\}$, where $p_{E}=f_{2_{E}} \tilde{\sim} g_{1_{E}}=\left\{p\left(e_{1}\right)=\{x / 0.5, y / 0.7\}, p\left(e_{2}\right)=\{x / 0.0, y / 0.6\}\right\}, q_{E}=f_{2_{E}} \tilde{\cup} g_{2_{E}}=$ $\left\{q\left(e_{1}\right)=\{x / 0.5, y / 0.7\}, q\left(e_{2}\right)=\{x / 0.3, y / 0.6\}\right\}$, while $f_{1_{E}} \tilde{\cup} g_{1_{E}}=f_{1_{E}} \tilde{\cup} g_{2_{E}}=g_{2_{E}}, f_{1_{E}} \tilde{\cup} g_{3_{E}}=g_{3_{E}}$. The $\tau_{1} \tau_{2}-f u z z y$ soft closed sets are $\left\{\tilde{1}_{\mathrm{E}}, \hat{g}_{1_{\mathrm{E}}}, \hat{f}_{1_{\mathrm{E}}}, \hat{f}_{2_{\mathrm{E}}}, \hat{\mathrm{p}}_{\mathrm{E}}, \hat{\mathrm{q}}_{\mathrm{E}}\right\}$, where

$$
\begin{aligned}
& \hat{f}_{1_{\mathrm{E}}}=\left\{\hat{f}_{1}\left(e_{1}\right)=\{x / 1.0, y / 1.0\}=\tilde{1}, \hat{f}_{1}\left(e_{2}\right)=\{x / 0.7, y / 1.0\}\right\}, \\
& \hat{f}_{2_{E}}=\left\{\hat{f}_{2}\left(e_{1}\right)=\{x / 1.0, y / 0.3\}, \hat{f}_{2}\left(e_{2}\right)=\{x / 1.0, y / 0.4\}\right\},
\end{aligned}
$$




$$
\begin{aligned}
\dot{g}_{1_{\mathrm{E}}} & =\left\{\dot{g}_{1}\left(e_{1}\right)=\{x / 0.5, y / 1.0\}, \dot{g}_{1}\left(e_{2}\right)=\{x / 1.0, y / 1.0\}=\tilde{1}\right\}, \\
\dot{g}_{2_{\mathrm{E}}} & =\left\{\dot{g}_{2}\left(e_{1}\right)=\{x / 0.5, y / 1.0\}, \dot{g}_{2}\left(e_{2}\right)=\{x / 0.7, y / 1.0\}\right\}, \\
\dot{g}_{3_{\mathrm{E}}} & =\left\{\dot{g}_{3}\left(e_{1}\right)=\{x / 0.5, y / 0.3\}, \dot{g}_{3}\left(e_{2}\right)=\{x / 0.7, y / 1.0\}\right\}, \\
\dot{p}_{\mathrm{E}} & =\left\{\dot{p}\left(e_{1}\right)=\{x / 0.5, y / 0.3\}, \hat{p}\left(e_{2}\right)=\{x / 1.0, y / 0.4\}\right\}, \\
\dot{q}_{\mathrm{E}} & =\left\{\dot{q}\left(e_{1}\right)=\{x / 0.5, y / 0.3\}, \hat{q}\left(e_{2}\right)=\{x / 0.7, y / 0.4\}\right\} .
\end{aligned}
$$

Let $h_{1_{\mathrm{E}}}, h_{2_{\mathrm{E}}} \tilde{\in} \widetilde{(X, E)}$, where $h_{1_{\mathrm{E}}}=\left\{h_{1}\left(e_{1}\right)=\{x / 0.5, y / 0.0\}, h_{1}\left(e_{2}\right)=\{x / 0.3, y / 0.0\}\right\}$ and $h_{2_{E}}=\left\{h_{2}\left(e_{1}\right)=\right.$ $\left.\{x / 0.0, y / 0.0\}=\tilde{0}, h_{2}\left(e_{2}\right)=\{x / 0.3, y / 0.6\}\right\}$,

(i) $\mathrm{g}_{2_{\mathrm{E}}}$ is a $(1,2)^{*}$-fuzzy soft open but not $(1,2)^{*}$-fuzzy soft regular open;

(ii) $h_{1_{\mathrm{E}}}$ is a $(1,2)^{*}$-fuzzy soft $\alpha$ open but not $(1,2)^{*}$-fuzzy soft open;

(iii) $h_{2_{\mathrm{E}}}$ is $a(1,2)^{*}$ is a $(1,2)^{*}$-fuzzy soft semi open but not $(1,2)^{*}$-fuzzy soft $\alpha$ open;

(iv) $h_{2_{\mathrm{E}}}$ is a $(1,2)^{*}$-fuzzy soft $\beta$ open set but not $(1,2)^{*}$-fuzzy soft preopen.

Example 3.5. Let $X=\{x, y\}, E=\left\{e_{1}, e_{2}\right\}$ and let $\tau_{1}=\left\{\tilde{0}_{E}, \tilde{1}_{E}, f_{E}\right\}, \tau_{2}=\left\{\tilde{0}_{E}, \tilde{1}_{E}, g_{E}\right\}$, where

$$
\begin{aligned}
f_{E} & =\left\{f\left(e_{1}\right)=\{x / 0.0, y / 0.7\}, f\left(e_{2}\right)=\{x / 0.0, y / 0.0\}=\tilde{0}\right\}, \\
g_{E} & =\left\{g\left(e_{1}\right)=\{x / 0.5, y / 0.0\}, g\left(e_{2}\right)=\{x / 0.0, y / 0.0\}=\tilde{0}\right\} .
\end{aligned}
$$

It is clear that $\left(X, E, \tau_{1}, \tau_{2}\right)$ is a fuzzy soft bitopological space. The $\tau_{1} \tau_{2}$-fuzzy soft open sets are $\left\{\tilde{0}_{\mathrm{E}}, \tilde{1}_{\mathrm{E}}, \mathrm{f}_{\mathrm{E}}, \mathrm{g}_{\mathrm{E}}, \mathrm{p}_{\mathrm{E}}\right\}$, where $\mathrm{p}_{\mathrm{E}}=\left\{\mathrm{p}\left(\mathrm{e}_{1}\right)=\{\mathrm{x} / 0.5, \mathrm{y} / 0.7\}, \mathrm{p}\left(\mathrm{e}_{2}\right)=\{\mathrm{x} / 0.0, \mathrm{y} / 0.0\}=\tilde{0}\right\}$. The $\tau_{1} \tau_{2}$-fuzzy soft closed sets are $\left\{\tilde{1}_{\mathrm{E}}, \tilde{1}_{\mathrm{E}}, \hat{f}_{\mathrm{E}}\right.$, $\left.\hat{g}_{\mathrm{E}}, \hat{\mathrm{p}}_{\mathrm{E}},\right\}$, where

$$
\begin{aligned}
& \hat{f}_{\mathrm{E}}=\left\{\hat{f}\left(e_{1}\right)=\{x / 1.0, y / 0.3\}, \hat{f}\left(e_{2}\right)=\{x / 1.0, y / 1.0\}=\tilde{1}\right\}, \\
& \dot{g}_{\mathrm{E}}=\left\{\dot{g}\left(e_{1}\right)=\{x / 0.5, y / 1.0\}, \dot{g}\left(e_{2}\right)=\{x / 1.0, y / 1.0\}=\tilde{1}\right\}, \\
& \dot{p}_{\mathrm{E}}=\left\{\dot{p}\left(e_{1}\right)=\{x / 0.5, y / 0.3\}, \dot{p}\left(e_{2}\right)=\{x / 1.0, y / 1.0\}=\tilde{1}\right\} .
\end{aligned}
$$

Let $h_{E} \tilde{E} \widetilde{(X, E)}$, where $h_{E}=\left\{h\left(e_{1}\right)=\{x / 0.4, y / 0.3\}, h\left(e_{2}\right)=\{x / 0.6, y / 0.3\}\right\}$.

(v) $h_{\mathrm{E}}$ is a $(1,2)^{*}$-fuzzy soft $\beta$-open set but not $(1,2)^{*}$-fuzzy soft semi open.

Example 3.6. Let $X=\{x, y, z, w\}, E=\left\{e_{1}, e_{2}, e_{3}\right\}$ and let $\tau_{1}=\left\{\tilde{0}_{E}, \tilde{1}_{E}, f_{1_{E}}, f_{2_{E}}, f_{3_{E}}, f_{4_{E}}, f_{5_{E}}, f_{6_{E}}, f_{7_{E}}, f_{8_{E}}, f_{9_{E}}\right.$, $\left.f_{10_{E}}, f_{11_{E}}, f_{12_{E}}, f_{13_{E}}, f_{14_{E}}, f_{15_{E}}\right\}, \tau_{2}=\left\{\tilde{0}_{E}, \tilde{1}_{E}\right\}$, where

$$
\begin{aligned}
f_{1_{\mathrm{E}}} & =\left\{f_{1}\left(e_{1}\right)=\{x / 2.0, y / 0.0, z / 0.1, w / 0.0\}, f_{1}\left(e_{2}\right)=\{x / 0.2, y / 0.1, z / 0.0, w / 0.0\},\right. \\
f_{1}\left(e_{3}\right) & =\{x / 0.2, y / 0.0, z / 0.0, w / 0.5\}\}, \\
f_{2_{\mathrm{E}}} & =\left\{f_{2}\left(e_{1}\right)=\{x / 0.0, y / 0.0, z / 0.4, w / 0.5\}, f_{2}\left(e_{2}\right)=\{x / 0.2, y / 0.0, z / 0.4, w / 0.5\},\right. \\
f_{2}\left(e_{3}\right) & =\{x / 0.2, y / 0.1, z / 0.0, w / 0.5\}\}, \\
f_{3_{\mathrm{E}}} & =\left\{f_{3}\left(e_{1}\right)=\{x / 0.0, y / 0.0, z / 0.0, w / 0.0\}=\tilde{0}\right\}, f_{3}\left(e_{2}\right)=\{x / 0.0, y / 0.0, z / 0.4, w / 0.0\}, \\
f_{3}\left(e_{3}\right) & =\{x / 0.2, y / 0.0, z / 0.0, w / 0.0\}\}, \\
f_{4 \mathrm{E}} & =\left\{f_{4}\left(e_{1}\right)=\{x / 0.2, y / 0.1, z / 0.0, w / 0.5\}, f_{4}\left(e_{2}\right)=\{x / 0.2, y / 0.1, z / 0.4, w / 0.5\},\right. \\
f_{4}\left(e_{3}\right) & =\{x / 0.2, y / 0.1, z / 0.4, w / 0.5\}\}, \\
f_{5_{\mathrm{E}}} & =\left\{f_{5}\left(e_{1}\right)=\{x / 0.2, y / 0.0, z / 0.4, w / 0.0\}, f_{5}\left(e_{2}\right)=\{x / 0.0, y / 0.1, z / 0.4, w / 0.0\},\right. \\
f_{5}\left(e_{3}\right) & =\{x / 0.2, y / 0.1, z / 0.4, w / 0.5\}\}, \\
f_{6_{\mathrm{E}}} & =\left\{f_{6}\left(e_{1}\right)=\{x / 0.2, y / 0.0, z / 0.0, w / 0.0\}, f_{6}\left(e_{2}\right)=\{x / 0.0, y / 0.1, z / 0.0, w / 0.0\},\right. \\
f_{6}\left(e_{3}\right) & =\{x / 0.0, y / 0.0, z / 0.0, w / 0.0\}=\tilde{0}\}, \\
f_{7 \mathrm{E}} & =\left\{f_{7}\left(e_{1}\right)=\{x / 0.2, y / 0.0, z / 0.4, w / 0.0\}, f_{7}\left(e_{2}\right)=\{x / 0.0, y / 0.1, z / 0.4, w / 0.5\},\right. \\
f_{7}\left(e_{3}\right) & =\{x / 0.2, y / 0.1, z / 0.0, w / 0.5\}\},
\end{aligned}
$$




$$
\begin{aligned}
f_{8_{\mathrm{E}}} & =\left\{f_{8}\left(e_{1}\right)=\{x / 0.0, y / 0.0, z / 0.0, w / 0.0\}=\tilde{0}\right\}, f_{8}\left(e_{2}\right)=\{x / 0.0, y / 0.0, z / 0.0, w / 0.5\}, \\
f_{8}\left(e_{3}\right) & =\{x / 0.0, y / 0.1, z / 0.0, w / 0.0\}\}, \\
f_{9_{E}} & =\left\{f_{9}\left(e_{1}\right)=\{x / 0.2, y / 0.1, z / 0.4, w / 0.5\}, f_{9}\left(e_{2}\right)=\{x / 0.0, y / 0.0, z / 0.0, w / 0.5\},\right. \\
f_{9}\left(e_{3}\right) & =\{x / 0.2, y / 0.1, z / 0.4, w / 0.0\}\}, \\
f_{10_{E}} & =\left\{f_{10}\left(e_{1}\right)=\{x / 0.2, y / 0.0, z / 0.4, w / 0.0\}, f_{10}\left(e_{2}\right)=\{x / 0.0, y / 0.1, z / 0.4, w / 0.5\},\right. \\
f_{10}\left(e_{3}\right) & =\{x / 0.2, y / 0.1, z / 0.0, w / 0.0\}\}, \\
f_{11_{E}} & =\left\{f_{11}\left(e_{1}\right)=\{x / 0.0, y / 0.1, z / 0.4, w / 0.5\}, f_{11}\left(e_{2}\right)=\{x / 0.2, y / 0.1, z / 0.4, w / 0.5\},\right. \\
f_{11}\left(e_{3}\right) & =\{x / 0.2, y / 0.1, z / 0.4, w / 0.0\}\}, \\
f_{12_{E}} & =\left\{f_{12}\left(e_{1}\right)=\{x / 0.2, y / 0.0, z / 0.0, w / 0.0\}, f_{12}\left(e_{2}\right)=\{x / 0.0, y / 0.1, z / 0.4, w / 0.5\},\right. \\
f_{12}\left(e_{3}\right) & =\{x / 0.2, y / 0.1, z / 0.0, w / 0.5\}\}, \\
f_{13_{E}} & =\left\{f_{13}\left(e_{1}\right)=\{x / 0.2, y / 0.0, z / 0.0, w / 0.0\}, f_{13}\left(e_{2}\right)=\{x / 0.0, y / 0.1, z / 0.0, w / 0.5\},\right. \\
f_{13}\left(e_{3}\right) & =\{x / 0.0, y / 0.1, z / 0.0, w / 0.0\}\}, \\
f_{14_{E}} & =\left\{f_{14}\left(e_{1}\right)=\{x / 0.0, y / 0.0, z / 0.4, w / 0.5\}, f_{14}\left(e_{2}\right)=\{x / 0.2, y / 0.1, z / 0.0, w / 0.0\},\right. \\
f_{14}\left(e_{3}\right) & =\{x / 0.0, y / 0.0, z / 0.0, w / 0.0\}=\tilde{0}\}, \\
f_{15_{E}} & =\left\{f_{15}\left(e_{1}\right)=\{x / 0.2, y / 0.0, z / 0.0, w / 0.0\}, f_{15}\left(e_{2}\right)=\{x / 0.0, y / 0.1, z / 0.4, w / 0.0\},\right. \\
f_{15}\left(e_{3}\right) & =\{x / 0.2, y / 0.0, z / 0.0, w / 0.0\}\} .
\end{aligned}
$$

It is clear that $\left(\mathrm{X}, \mathrm{E}, \tau_{1}, \tau_{2}\right)$ is a fuzzy soft bitopological space.

(vi) Consider $\mathrm{f}_{\mathrm{E}} \tilde{\in} \widetilde{(X, E)}$, where $\mathrm{f}_{\mathrm{E}}=\left\{f\left(e_{1}\right)=\{x / 0.0, y / 0 ., z / 0.0, w / 0.7\}, f\left(e_{2}\right)=\{x / 0.0, y / 0.2, z / 0.5, w / 0.0\}\right.$, $\left.f\left(e_{3}\right)=\{x / 0.3, y / 0.0, z / 0.0, w / 0.0\}\right\}, \tau_{1} \tau_{2} \operatorname{int}\left(\tau_{1} \tau_{2} c l\left(f_{E}\right)\right)=\tilde{1}$ and $f_{E} \tilde{\subseteq} \tilde{1}_{\mathrm{E}}$. But

$$
\tau_{1} \tau_{2} \operatorname{int}\left(\tau_{1} \tau_{2} \operatorname{cl}\left(\tau_{1} \tau_{2} \operatorname{int}\left(f_{E}\right)\right)\right)=\tilde{0_{E}} .
$$

Hence, $\mathrm{f}_{\mathrm{E}}$ is $(1,2)^{*}$-fuzzy soft pre open set but not $(1,2)^{*}$-fuzzy soft $\alpha$-open.

Definition 3.7. Let $\left(X, E, \tau_{1}, \tau_{2}\right)$ be a fuzzy soft bitopological space and $f_{E} \widetilde{E} \widetilde{(X, E)}$. Then $f_{E}$ is called $(1,2)^{*}$ fuzzy soft b-open set (briefly, $(1,2)^{*}$-fsb-open) if $f_{E} \tilde{\subseteq} \tau_{1} \tau_{2} \operatorname{int}\left(\tau_{1} \tau_{2} \operatorname{cl}\left(f_{E}\right)\right) \tilde{\sim} \tau_{1} \tau_{2} c l\left(\tau_{1} \tau_{2} \operatorname{int}\left(f_{E}\right)\right)$.

Example 3.8. The fuzzy soft sets $\tilde{0}_{E}, \tilde{1}_{E}, g_{1_{E}}, g_{2_{E}}, g_{3_{E}}, g_{4_{E}}, g_{5_{E}}, g_{6_{E}}, g_{7_{E}}, g_{8_{E}}, g_{9_{E}} \tilde{\in} \widetilde{(X, E)}$ in Example 3.4, where

$$
\begin{aligned}
& g_{1_{\mathrm{E}}}=\left\{g_{1}\left(e_{1}\right)=\{x / 0.2, y / 0.0\}, g_{1}\left(e_{2}\right)=\{x / 0.0, y / 0.0\}=\tilde{0}\right\}, \\
& g_{2_{\mathrm{E}}}=\left\{g_{2}\left(e_{1}\right)=\{x / 0.0, y / 0.0\}=\tilde{0}, g_{2}\left(e_{2}\right)=\{x / 0.5, y / 0.0\}\right\}, \\
& g_{3_{\mathrm{E}}}=\left\{g_{3}\left(e_{1}\right)=\{x / 0.0, y / 0.0\}=\tilde{0}, g_{3}\left(e_{2}\right)=\{x / 0.5, y / 0.7\}\right\}, \\
& g_{4_{\mathrm{E}}}=\left\{g_{4}\left(e_{1}\right)=\{x / 0.2, y / 0.0\}, g_{4}\left(e_{2}\right)=\{x / 0.5, y / 0.0\}\right\}, \\
& g_{5_{\mathrm{E}}}=\left\{g_{5}\left(e_{1}\right)=\{x / 0.2, y / 0.0\}, g_{6}\left(e_{2}\right)=\{x / 0.0, y / 0.7\}\right\}, \\
& g_{6_{\mathrm{E}}}=\left\{g_{6}\left(e_{1}\right)=\{x / 0.2, y / 0.0\}, g_{3}\left(e_{2}\right)=\{x / 0.5, y / 0.7\}\right\}, \\
& g_{7_{\mathrm{E}}}=\left\{g_{7}\left(e_{1}\right)=\{x / 0.0, y / 0.6\}, g_{7}\left(e_{2}\right)=\{x / 0.5, y / 0.0\}\right\}, \\
& g_{8_{\mathrm{E}}}=\left\{g_{8}\left(e_{1}\right)=\{x / 0.0, y / 0.6\}, g_{8}\left(e_{2}\right)=\{x / 0.0, y / 0.7\}\right\}, \\
& g_{9_{\mathrm{E}}}=\left\{g_{9}\left(e_{1}\right)=\{x / 0.2, y / 0.6\}, g_{9}\left(e_{2}\right)=\{x / 0.5, y / 0.0\}\right\},
\end{aligned}
$$

are $(1,2)^{*}$-fuzzy soft b-open sets.

Theorem 3.9. Let $\left(\mathrm{X}, \mathrm{E}, \tau_{1}, \tau_{2}\right)$ be a fuzzy soft bitopological space. Then

(i) every $(1,2)^{*}$-fuzzy soft pre-open set is $(1,2)^{*}$-fuzzy soft b-open set;

(ii) every $(1,2)^{*}$-fuzzy soft b-open set is $(1,2)^{*}$-fuzzy soft $\beta$-open set;

(iii) every $(1,2)^{*}$-fuzzy soft semi open set is $(1,2)^{*}$-fuzzy soft b-open set. 
Proof.

(i). Let $f_{E}$ be a $(1,2)^{*}$-fuzzy soft pre-open set. Then

$$
f_{E} \tilde{\subseteq} \tau_{1} \tau_{2} \operatorname{int}\left(\tau_{1} \tau_{2} c l\left(f_{E}\right)\right) \tilde{\subseteq} \tau_{1} \tau_{2} \operatorname{int}\left(\tau_{1} \tau_{2} c l\left(f_{E}\right)\right) \tilde{\cup} \tau_{1} \tau_{2} \operatorname{int}\left(f_{E}\right) \tilde{\subseteq} \tau_{1} \tau_{2} \operatorname{int}\left(\tau_{1} \tau_{2} c l\left(f_{E}\right)\right) \tilde{U} \tau_{1} \tau_{2} c l\left(\tau_{1} \tau_{2} \operatorname{int}\left(f_{E}\right)\right),
$$

which implies to $f_{E}$ is $(1,2)^{*}$-fuzzy soft b-open set.

(ii). Let $f_{E}$ be a $(1,2)^{*}$-fuzzy soft b-open set. Then

$$
f_{E} \tilde{\subseteq} \tau_{1} \tau_{2} \operatorname{cl}\left(f_{E}\right) \tilde{\cup} \tau_{1} \tau_{2} \operatorname{int}\left(\tau_{1} \tau_{2} \operatorname{cl}\left(f_{E}\right)\right) \tilde{\subseteq} \tau_{1} \tau_{2} \operatorname{cl}\left(\tau_{1} \tau_{2} \operatorname{int}\left(\tau_{1} \tau_{2} \operatorname{cl}\left(f_{E}\right)\right)\right) \tilde{\cup}\left(\tau_{1} \tau_{2} \operatorname{int}\left(\tau_{1} \tau_{2} \operatorname{cl}\left(f_{E}\right)\right)\right) .
$$

Thus $f_{E}$ is $(1,2)^{*}$-fuzzy soft $\beta$-open set.

(iii). Let $f_{E}$ be a $(1,2)^{*}$-fuzzy soft semi open set. This implies

$$
f_{E} \tilde{\subseteq} \tau_{1} \tau_{2} c l\left(\tau_{1} \tau_{2} \operatorname{int}\left(f_{E}\right)\right) \tilde{\subseteq} \tau_{1} \tau_{2} c l\left(\tau_{1} \tau_{2} \operatorname{int}\left(f_{E}\right)\right) \tilde{U} \tau_{1} \tau_{2} c l\left(f_{E}\right) \tilde{\subseteq} \tau_{1} \tau_{2} c l\left(\tau_{1} \tau_{2} \operatorname{int}\left(f_{E}\right)\right) \tilde{\cup} \tau_{1} \tau_{2} \operatorname{int}\left(\tau_{1} \tau_{2} c l\left(f_{E}\right)\right) .
$$

Thus $f_{E}$ is $(1,2)^{*}$-fuzzy soft b-open set.

Remark 3.10. The converse of the above theorem is need not be true as shown in the following example.

Example 3.11. Let $\left(\mathrm{X}, \mathrm{E}, \tau_{1}, \tau_{2}\right)$ be a fuzzy soft bitopological space, where $\mathrm{X}, \mathrm{E}, \tau_{1}, \tau_{2}$ are given as in Example 3.5.

(i) The fuzzy soft set $\mathrm{f}_{\mathrm{E}}=\left\{\mathrm{f}\left(\mathrm{e}_{1}\right)=\{x / 0.5, y / 0.0\}, f\left(e_{2}\right)=\{x / 0.3, y / 0.0\}\right\} \tilde{\in} \widetilde{(X, E)}$ is $(1,2)^{*}$-fuzzy soft b-open set but not $(1,2)^{*}$-fuzzy soft pre-open set.

(ii) The fuzzy soft set $g_{\mathrm{E}}=\left\{\mathrm{g}\left(\mathrm{e}_{1}\right)=\{x / 0.0, \mathrm{y} / 0.0\}=\tilde{0}, \mathrm{~g}\left(\mathrm{e}_{2}\right)=\{x / 0.0, \mathrm{y} / 0.6\}\right\} \widetilde{\mathrm{E}(\mathrm{X}, \mathrm{E})}$ is $(1,2)^{*}$-fuzzy soft $\beta$-open set but not $(1,2)^{*}$-fuzzy soft b-open set.

(iii) The fuzzy soft set $\mathrm{h}_{\mathrm{E}}=\left\{\mathrm{h}\left(\mathrm{e}_{1}\right)=\{x / 0.0, y / 0.0\}=\tilde{0}, \mathrm{~h}\left(e_{2}\right)=\{x / 0.3, y / 0.0\}\right\} \widetilde{\in(X, E)}$ is $(1,2)^{*}$-fuzzy soft b-open set but not $(1,2)^{*}$-fuzzy soft semi open set.

Remark 3.12. The above discussions are summarized in the following diagram:

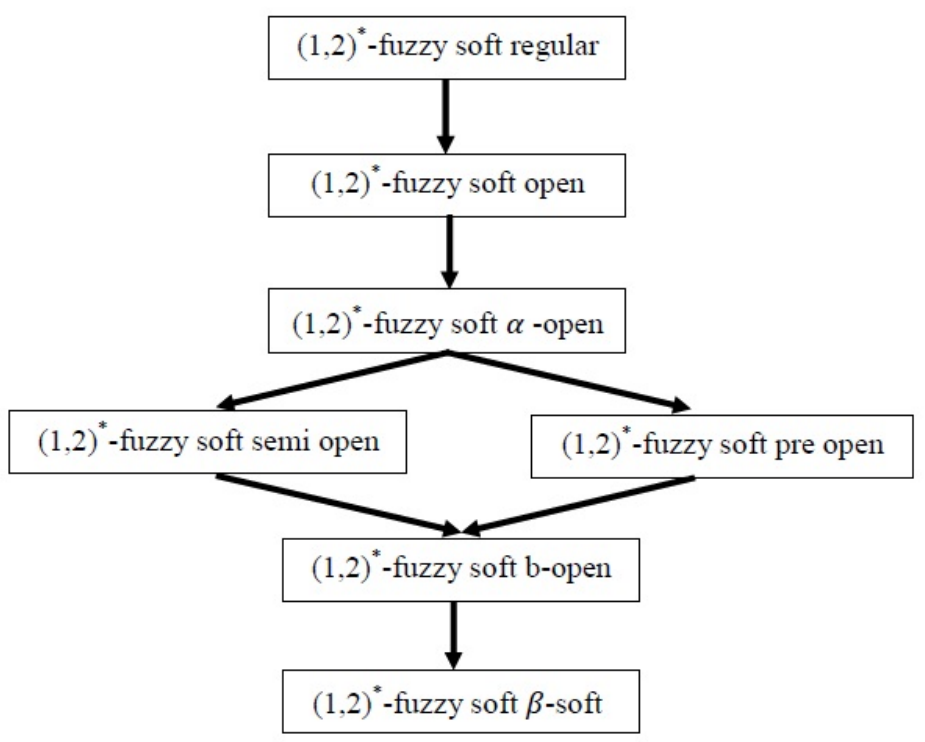

Theorem 3.13. An arbitrary union of $(1,2)^{*}$-fuzzy soft b-open sets are $(1,2)^{*}$-fuzzy soft b-open set. 
Proof. Let $\left\{\left(f_{E}\right)_{i}\right\}$ be a collection of $(1,2)^{*}$-fuzzy softb-open sets. Then for each $i$,

$$
\begin{aligned}
& \left(f_{E}\right)_{i} \tilde{\widetilde{C}} \tau_{1} \tau_{2} \operatorname{int}\left(\tau_{1} \tau_{2} \operatorname{cl}\left(\left(f_{E}\right)_{i}\right)\right) \tilde{U} \tau_{1} \tau_{2} \operatorname{cl}\left(\tau_{1} \tau_{2} \operatorname{int}\left(\left(f_{E}\right)_{i}\right)\right) \tilde{\cup}_{i}\left(\left(f_{E}\right)_{i}\right) \\
& \tilde{\subseteq} \cup_{i}\left\{\tau_{1} \tau_{2} \operatorname{int}\left(\tau_{1} \tau_{2} \operatorname{cl}\left(\left(f_{E}\right)_{i}\right)\right) \tilde{\cup} \tau_{1} \tau_{2} \operatorname{cl}\left(\left(f_{E}\right)_{i}\right)\right\} \\
& \tilde{\subseteq}\left[U_{i}\left\{\tau_{1} \tau_{2} \operatorname{int}\left(\tau_{1} \tau_{2} \operatorname{cl}\left(\left(f_{E}\right)_{i}\right)\right)\right\}\right] \cup\left[U_{i}\left\{\tau_{1} \tau_{2} \operatorname{cl}\left(\tau_{1} \tau_{2} \operatorname{int}\left(\left(f_{E}\right)_{i}\right)\right)\right\}\right] \\
& \tilde{\subseteq}\left[\tau _ { 1 } \tau _ { 2 } \operatorname { i n t } \{ \tilde { U } _ { i } ( \tau _ { 1 } \tau _ { 2 } \operatorname { c l } ( ( f _ { E } ) _ { i } ) \} ] \tilde { C } \left[\tau_{1} \tau_{2} \operatorname{cl}\left\{\tilde{U}_{i}\left(\tau_{1} \tau_{2} \operatorname{int}\left(\left(f_{E}\right)_{i}\right)\right\}\right]\right.\right. \\
& \tilde{\subseteq}\left[\tau _ { 1 } \tau _ { 2 } \operatorname { i n t } \{ ( \tau _ { 1 } \tau _ { 2 } \operatorname { c l } \{ \cup _ { i } ( ( f _ { E } ) _ { i } ) \} ] ] \tilde { \cup } \left[\tau_{1} \tau_{2} \operatorname{cl}\left\{\left(\tau_{1} \tau_{2} \operatorname{int}\left\{\cup_{i}\left(\left(f_{E}\right)_{i}\right)\right\}\right\}\right] .\right.\right.
\end{aligned}
$$

Hence the theorem is proved.

Remark 3.14. The intersection of two $(1,2)^{*}$-fuzzy soft b-open sets need not be $(1,2)^{*}$-fuzzy soft b-open set. In example 3.8, $f_{3_{E}} \tilde{\cap} f_{5_{E}}=h_{E}=\left\{h_{E}\left(e_{1}\right)=\{x / 0.0, y / 0.0\}=0, h_{E}\left(e_{2}\right)=\{x / 0.0, y / 0.7\}\right\}$, which is not $(1,2)^{*}$-fuzzy soft b-open set.

Theorem 3.15. Let $\left(\mathrm{X}, \mathrm{E}, \tau_{1}, \tau_{2}\right)$ be a fuzzy soft bitopological space. Then

(i) the intersection of $(1,2)^{*}$-fuzzy soft open set and $(1,2)^{*}$-fsb-open set is $(1,2)^{*}$-fsb-open set;

(ii) the intersection of $(1,2)^{*}$-fs $\alpha$-open set and $(1,2)^{*}$-fsb-open set is $(1,2)^{*}$-fsb-open set.

Proof.

(i). Let $f_{E}$ be a $(1,2)^{*}$-fuzzy soft open set and $g_{E}$ be a $(1,2)^{*}$-fsb-open set, then

$$
\begin{aligned}
& f_{E} \tilde{\cap} g_{E} \tilde{\subseteq} f_{E} \tilde{\cap}\left[\tau_{1} \tau_{2} \operatorname{cl}\left(\tau_{1} \tau_{2} \operatorname{int}\left(g_{E}\right)\right) \tilde{\cup} \tau_{1} \tau_{2} \operatorname{int}\left(\tau_{1} \tau_{2} \operatorname{cl}\left(g_{E}\right)\right)\right] \\
& \quad=f_{E} \tilde{\cap}\left[\tau_{1} \tau_{2} \operatorname{cl}\left(\tau_{1} \tau_{2} \operatorname{int}\left(g_{E}\right)\right)\right] \tilde{\cup}\left[f_{E} \tilde{\cap}\left[\tau_{1} \tau_{2} \operatorname{int}\left(\tau_{1} \tau_{2} \operatorname{cl}\left(g_{E}\right)\right)\right]\right. \\
& \quad \tilde{\subseteq}\left[\tau_{1} \tau_{2} c l\left(\tau_{1} \tau_{2} \operatorname{int}\left(f_{E}\right)\right) \tilde{\cap} \tau_{1} \tau_{2} c l\left(\tau_{1} \tau_{2} \operatorname{int}\left(g_{E}\right)\right)\right] \cup \tilde{\cup}\left[\tau_{1} \tau_{2} \operatorname{int}\left(\tau_{1} \tau_{2} \operatorname{cl}\left(f_{E}\right)\right) \tilde{\cap} \tau_{1} \tau_{2} \operatorname{int}\left(\tau_{1} \tau_{2} c l\left(g_{E}\right)\right)\right] \\
& \quad \tilde{\subseteq}\left[\tau_{1} \tau_{2} \operatorname{cl}\left(\tau_{1} \tau_{2} \operatorname{int}\left(f_{E} \tilde{\cap} g_{E}\right)\right)\right] \tilde{\cup}\left[\tau_{1} \tau_{2} \operatorname{int}\left(\tau_{1} \tau_{2} \operatorname{cl}\left(f_{E} \tilde{\cap} g_{E}\right)\right)\right] .
\end{aligned}
$$

Thus $f_{E} \tilde{\cap} g_{E}$ is $(1,2)^{*}$-fsb-open set.

(ii). Let $f_{E}$ be a $(1,2)^{*}$-fuzzy soft $\alpha$-open set and $g_{E}$ be a $(1,2)^{*}$-fsb-open set, then

$$
\begin{aligned}
f_{E} \tilde{\cap} g_{E} & \tilde{\subseteq}\left[\tau_{1} \tau_{2} \operatorname{int}\left(\tau_{1} \tau_{2} \operatorname{cl}\left(\tau_{1} \tau_{2} \operatorname{int}\left(f_{E}\right)\right)\right)\right] \tilde{\cap}\left[\tau_{1} \tau_{2} \operatorname{cl}\left(\tau_{1} \tau_{2} \operatorname{int}\left(g_{E}\right)\right) \tilde{\cup} \tau_{1} \tau_{2} \operatorname{int}\left(\tau_{1} \tau_{2} \operatorname{cl}\left(g_{E}\right)\right)\right] \\
& =\left[\tau_{1} \tau_{2} \operatorname{int}\left(\tau_{1} \tau_{2} \operatorname{cl}\left(\tau_{1} \tau_{2} \operatorname{int}\left(f_{E}\right)\right)\right) \tilde{\cap} \tau_{1} \tau_{2} \operatorname{cl}\left(\tau_{1} \tau_{2} \operatorname{int}\left(g_{E}\right)\right)\right] \tilde{\cup}\left[\tau_{1} \tau_{2} \operatorname{int}\left(\tau_{1} \tau_{2} \operatorname{cl}\left(f_{E}\right)\right) \tilde{n} \tau_{1} \tau_{2} \operatorname{int}\left(\tau_{1} \tau_{2} \operatorname{cl}\left(g_{E}\right)\right)\right] \\
& \tilde{\subseteq}\left[\tau_{1} \tau_{2} \operatorname{cl}\left(\tau_{1} \tau_{2} \operatorname{int}\left(f_{E} \tilde{\cap} g_{E}\right)\right)\right] \tilde{\cup}\left[\tau_{1} \tau_{2} \operatorname{int}\left(\tau_{1} \tau_{2} \operatorname{cl}\left(f_{E} \tilde{\cap} g_{E}\right)\right)\right] .
\end{aligned}
$$

Thus $f_{E} \tilde{\cap} g_{E}$ is $(1,2)^{*}$-fsb-open set.

Theorem 3.16. Any $(1,2)^{*}-\mathrm{fsb}$-open set $\mathrm{f}_{\mathrm{E}}$ in fuzzy soft bitopological space $\left(\mathrm{X}, \mathrm{E}, \tau_{1}, \tau_{2}\right), \tau_{1} \tau_{2} \mathrm{cl}\left(\mathrm{f}_{\mathrm{E}}\right)$ is a $(1,2)^{*}$ fsr-closed set.

Proof. Let $f_{E}$ be a $(1,2)^{*}$-fsb-open set, this implies $f_{E} \tilde{\subseteq} \tau_{1} \tau_{2} c l\left(\tau_{1} \tau_{2} \operatorname{int}\left(f_{E}\right)\right) \tilde{U} \tau_{1} \tau_{2} \operatorname{int}\left(\tau_{1} \tau_{2} \operatorname{cl}\left(f_{E}\right)\right)$. Also

$$
\tau_{1} \tau_{2} \operatorname{cl}\left(f_{E}\right) \tilde{\simeq} \tau_{1} \tau_{2} \operatorname{cl}\left(\tau_{1} \tau_{2} \operatorname{cl}\left(\tau_{1} \tau_{2} \operatorname{int}\left(f_{E}\right)\right)\right) \tilde{U} \tau_{1} \tau_{2} \operatorname{cl}\left(\tau_{1} \tau_{2} \operatorname{int}\left(\tau_{1} \tau_{2} \operatorname{cl}\left(f_{E}\right)\right)\right) \tilde{\subseteq} \tau_{1} \tau_{2} \operatorname{cl}\left(\tau_{1} \tau_{2} \operatorname{int}\left(\tau_{1} \tau_{2} \operatorname{cl}\left(f_{E}\right)\right)\right)
$$

and $\tau_{1} \tau_{2} \operatorname{cl}\left(\tau_{1} \tau_{2} \operatorname{int}\left(\tau_{1} \tau_{2} \operatorname{cl}\left(f_{E}\right)\right)\right) \tilde{\subseteq} \tau_{1} \tau_{2} c l\left(f_{E}\right)$. Therefore $\tau_{1} \tau_{2} \operatorname{cl}\left(f_{E}\right)=\tau_{1} \tau_{2} \operatorname{cl}\left(\tau_{1} \tau_{2} \operatorname{int}\left(\tau_{1} \tau_{2} \operatorname{cl}\left(f_{E}\right)\right)\right)$. So $\tau_{1} \tau_{2} c l\left(f_{E}\right)$ is a $(1,2)^{*}$-fsr-closed set.

Theorem 3.17. Let $\mathrm{f}_{\mathrm{E}}$ be a $(1,2)^{*}$-fsb-open set in fuzzy soft bitopological space $\left(\mathrm{X}, \mathrm{E}, \tau_{1}, \tau_{2}\right)$.

(i) If $\tau_{1} \tau_{2} \mathrm{cl}\left(\mathrm{f}_{\mathrm{E}}\right)=\tilde{0}_{\mathrm{E}}$, then $\mathrm{f}_{\mathrm{E}}$ is a $(1,2)^{*}$-fss-open set.

(ii) If $\tau_{1} \tau_{2} \operatorname{int}\left(f_{\mathrm{E}}\right)=\tilde{0}_{\mathrm{E}}$, then $\mathrm{f}_{\mathrm{E}}$ is $a(1,2)^{*}$-fsp-open set. 
Proof.

(i). Let $f_{E}$ be a $f_{E}(1,2)^{*}$-fsb-open set, this implies $f_{E} \tilde{\subseteq} \tau_{1} \tau_{2} c l\left(\tau_{1} \tau_{2} \operatorname{int}\left(f_{E}\right)\right) \tilde{U} \tau_{1} \tau_{2} \operatorname{int}\left(\tau_{1} \tau_{2} c l\left(f_{E}\right)\right)$. Since $\tau_{1} \tau_{2} \operatorname{cl}\left(f_{E}\right)=\tilde{0}_{E}, f_{E} \tilde{\subseteq} \tau_{1} \tau_{2} c l\left(\tau_{1} \tau_{2} \operatorname{int}\left(f_{E}\right)\right)$. Thus $f_{E}$ is a $(1,2)^{*}$-fss-open set.

(ii). Since $\tau_{1} \tau_{2} \operatorname{int}\left(f_{E}\right)=\tilde{0}_{E}, f_{E} \tilde{\subseteq} \tau_{1} \tau_{2} \operatorname{int}\left(\tau_{1} \tau_{2} c l\left(f_{E}\right)\right)$. Thus $f_{E}$ is a $(1,2)^{*}$-fsp-open set.

Theorem 3.18. Let $\mathrm{f}_{\mathrm{E}}$ be a $(1,2)^{*}$-fsb-open set in fuzzy soft bitopological space $\left(\mathrm{X}, \mathrm{E}, \tau_{1}, \tau_{2}\right)$.

(i) If $\mathrm{f}_{\mathrm{E}}$ is $(1,2)^{*}$-fsr-closed set, then $\mathrm{f}_{\mathrm{E}}$ is $a(1,2)^{*}$-fsp-open set.

(ii) If $\mathrm{f}_{\mathrm{E}}$ is $\mathrm{f}_{\mathrm{E}}(1,2)^{*}$-fsp-open set, then $\mathrm{f}_{\mathrm{E}}$ is $(1,2)^{*}$-fss-open set.

Proof.

(i). Since $f_{E}$ be a $(1,2)^{*}$-fsb-open set, $f_{E} \tilde{\subseteq} \tau_{1} \tau_{2} c l\left(\tau_{1} \tau_{2} \operatorname{int}\left(f_{E}\right)\right) \tilde{\cup} \tau_{1} \tau_{2} \operatorname{int}\left(\tau_{1} \tau_{2} c l\left(f_{E}\right)\right)$. Let $f_{E}$ be a $(1,2)^{*}$ fsr-closed set, $f_{E}=\tau_{1} \tau_{2} \operatorname{cl}\left(\tau_{1} \tau_{2} \operatorname{int}\left(f_{E}\right)\right)$. Then $f_{E} \tilde{\subseteq} f_{E} \tilde{\cup} \tau_{1} \tau_{2} \operatorname{int}\left(\tau_{1} \tau_{2} c l\left(f_{E}\right)\right)$. Thus $f_{E}$ is a $(1,2)^{*}$-fsp-open set.

(ii). Let $f_{E}$ be a $(1,2)^{*}$-fsr-open set, $f_{E}=\tau_{1} \tau_{2} \operatorname{int}\left(\tau_{1} \tau_{2} \operatorname{cl}\left(f_{E}\right)\right) . f_{E} \tilde{\subseteq} f_{E} \tilde{\cup} \tau_{1} \tau_{2} \operatorname{cl}\left(\tau_{1} \tau_{2} \operatorname{int}\left(f_{E}\right)\right)$ which implies, $f_{E} \tilde{\subseteq} \tau_{1} \tau_{2} c l\left(\tau_{1} \tau_{2} \operatorname{int}\left(f_{E}\right)\right)$. Thus $f_{E}$ is a $(1,2)^{*}$-fss-open set.

Theorem 3.19. Let $\mathrm{f}_{\mathrm{E}}$ be a $(1,2)^{*}$-fsb-open set in fuzzy soft bitopological space $\left(\mathrm{X}, \mathrm{E}, \tau_{1}, \tau_{2}\right)$ if and only if $\mathrm{f}_{\mathrm{E}}$ is the union of $(1,2)^{*}$-fss-open set and $(1,2)^{*}$-fsp-open set.

Proof. Follows from the definition of $(1,2)^{*}$-fsb-open set in fuzzy soft bitopological space $\left(X, E, \tau_{1}, \tau_{2}\right)$.

\section{4. $(1,2)^{*}$-Fuzzy Soft b-closed sets}

In this section we introduce $(1,2)^{*}$-fuzzy soft b-closed sets in fuzzy soft bitopological spaces and study some of their properties.

Definition 4.1. Let $\left(X, E, \tau_{1}, \tau_{2}\right)$ be a fuzzy soft bitopological space and $f_{E} \tilde{E} \widetilde{(X, E)}$. Then $f_{E}$ is called $(1,2)^{*}$-fuzzy soft b-closed set (briefly, $(1,2)^{*}$-fsb-closed) if $\tau_{1} \tau_{2} \operatorname{int}\left(\tau_{1} \tau_{2} c l\left(f_{E}\right)\right) \tilde{U} \tau_{1} \tau_{2} c l\left(\tau_{1} \tau_{2} \operatorname{int}\left(f_{E}\right)\right) \tilde{\subseteq} f_{E}$. The complement of $(1,2)^{*}$-fuzzy soft b-closed set is $(1,2)^{*}$-fuzzy soft b-open set.

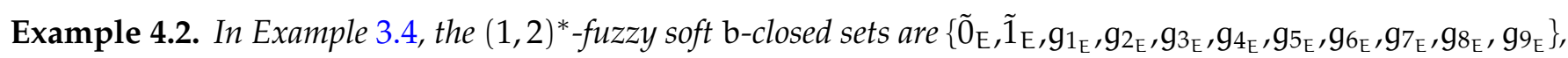
where

$$
\begin{aligned}
& \dot{g}_{1_{\mathrm{E}}}=\left\{\dot{g}_{1}\left(e_{1}\right)=\{x / 0.8, y / 1.0\}, \dot{g}_{1}\left(e_{2}\right)=\{x / 1.0, y / 1.0\}=\tilde{1}\right\}, \\
& \dot{g}_{2_{\mathrm{E}}}=\left\{\dot{g}_{2}\left(e_{1}\right)=\{x / 1.0, y / 1.0\}=\tilde{1}, \dot{g}_{2}\left(e_{2}\right)=\{x / 0.5, y / 1.0\}\right\} \text {, } \\
& \dot{g}_{3_{\mathrm{E}}}=\left\{\dot{g}_{3}\left(e_{1}\right)=\{x / 1.0, y / 1.0\}=\tilde{1}, \dot{g}_{3}\left(e_{2}\right)=\{x / 0.5, y / 0.3\}\right\} \text {, } \\
& \dot{g}_{4_{\mathrm{E}}}=\left\{\dot{g}_{4}\left(e_{1}\right)=\{x / 0.8, y / 1.0\}, \dot{g}_{4}\left(e_{2}\right)=\{x / 0.5, y / 1.0\}\right\}, \\
& \dot{g}_{5_{\mathrm{E}}}=\left\{\dot{g}_{5}\left(e_{1}\right)=\{x / 0.8, y / 1.0\}, \dot{g}_{6}\left(e_{2}\right)=\{x / 1.0, y / 0.3\}\right\}, \\
& \dot{g}_{6_{\mathrm{E}}}=\left\{\dot{g}_{6}\left(e_{1}\right)=\{x / 0.8, y / 1.0\}, \dot{g}_{3}\left(e_{2}\right)=\{x / 0.5, y / 0.3\}\right\}, \\
& \dot{g}_{7_{\mathrm{E}}}=\left\{\hat{g}_{7}\left(e_{1}\right)=\{x / 1.0, y / 0.4\}, \hat{g}_{7}\left(e_{2}\right)=\{x / 0.5, y / 1.0\}\right\}, \\
& \mathfrak{g}_{8_{\mathrm{E}}}=\left\{\hat{g}_{8}\left(e_{1}\right)=\{x / 1.0, y / 0.4\}, \mathfrak{g}_{8}\left(e_{2}\right)=\{x / 1.0, y / 0.3\}\right\}, \\
& \dot{g}_{9_{\mathrm{E}}}=\left\{\dot{g}_{9}\left(e_{1}\right)=\{x / 0.8, y / 0.4\}, \dot{g}_{9}\left(e_{2}\right)=\{x / 0.5, y / 1.0\}\right\} \text {. }
\end{aligned}
$$

Theorem 4.3. An arbitrary intersection of $(1,2)^{*}$-fuzzy soft b-closed sets is a $(1,2)^{*}$-fuzzy soft b-closed set.

Proof. Let $\left\{\left(f_{E}\right)_{i}\right\}$ be a collection of $(1,2)^{*}$-fuzzy softb-closed sets in $\left(X, E, \tau_{1}, \tau_{2}\right)$. Then for each $i$,

$$
\tau_{1} \tau_{2} \operatorname{int}\left(\tau_{1} \tau_{2} \operatorname{cl}\left(\left(f_{E}\right)_{i}\right)\right) \tilde{\cup} \tau_{1} \tau_{2} \operatorname{cl}\left(\tau_{1} \tau_{2} \operatorname{int}\left(\left(f_{E}\right)_{i}\right)\right) \tilde{\subseteq}\left(f_{E}\right)_{i} .
$$

Since $\left\{\left(f_{E}\right)_{i}^{c}\right\}$ is an arbitrary family of $(1,2)^{*}$-open sets in $\left(X, E, \tau_{1}, \tau_{2}\right)$, hence, by Theorem 3.13, $\tilde{\cup}\left(f_{E}\right)_{i}^{c}$ is $(1,2)^{*}$-fuzzy softb-open set. But $\tilde{\cup}\left(f_{E}\right)_{i}^{c}=\left[\tilde{\cap}_{i}\left(f_{E}\right)_{i}\right]^{c}$. Therefore $\tilde{\cap}_{i}\left(f_{E}\right)_{i}$ is a $(1,2)^{*}$-fuzzy soft b-closed set. 
Remark 4.4. The union of two $(1,2)^{*}$-fuzzy soft b-closed sets need not be $(1,2)^{*}$-fuzzy soft b-closed set. In Example 3.11, $\hat{f}_{E}=\left\{\hat{f}\left(e_{1}\right)=\{x / 0.5, y / 1.0\}, f\left(e_{2}\right)=\{x / 0.7, y / 0.1\}\right\}$ and $\hat{h}_{E}=\left\{\hat{h}\left(e_{1}\right)=\{x / 1.0, y / 1.0\}=\right.$ $\left.\tilde{1}, \hat{h}\left(e_{2}\right)=\{x / 0.7, y / 0.1\}\right\}$ are $(1,2)^{*}$-fuzzy softb-closed sets. But the union is not $(1,2)^{*}$-fuzzy soft b-open set. $\hat{f}_{\mathrm{E}} \tilde{\cup} \mathfrak{h}\left(e_{1}\right)=\mathrm{k}_{\mathrm{E}}=\left\{\hat{k}\left(e_{1}\right)=\{x / 0.5, y / 1.0\}, k\left(e_{2}\right)=\{x / 0.7, y / 0.1\}\right\}$, which is not $(1,2)^{*}$-fuzzy soft b-closed set.

Theorem 4.5. Let $\mathrm{f}_{\mathrm{E}}$ be a $(1,2)^{*}$-fsb-closed set in fuzzy soft bitopological space $\left(\mathrm{X}, \mathrm{E}, \tau_{1}, \tau_{2}\right)$.

(i) If $\mathrm{f}_{\mathrm{E}}$ is $(1,2)^{*}$-fsr-closed set, then $\mathrm{f}_{\mathrm{E}}$ is a $(1,2)^{*}$-fss-closed set.

(ii) If $\mathrm{f}_{\mathrm{E}}$ is $\mathrm{f}_{\mathrm{E}}(1,2)^{*}$-fsp-open set, then $\mathrm{f}_{\mathrm{E}}$ is $(1,2)^{*}$-fsp-closed set.

Proof.

(i). Since $f_{E}$ be a $(1,2)^{*}$-fsb-closed set, $\tau_{1} \tau_{2} c l\left(\tau_{1} \tau_{2} \operatorname{int}\left(f_{E}\right)\right) \tilde{n} \tau_{1} \tau_{2} \operatorname{int}\left(\tau_{1} \tau_{2} c l\left(f_{E}\right)\right) \tilde{\subseteq} f_{E}$. Since $f_{E}$ be a $(1,2)^{*}$ fsr-closed set, $f_{E}=\tau_{1} \tau_{2} c l\left(\tau_{1} \tau_{2} \operatorname{int}\left(f_{E}\right)\right)$. Therefore $f_{E} \tilde{\cap} \tau_{1} \tau_{2} \operatorname{int}\left(\tau_{1} \tau_{2} c l\left(f_{E}\right)\right) \tilde{\subseteq} f_{E}$. Thus,

$$
\tau_{1} \tau_{2} \operatorname{int}\left(\tau_{1} \tau_{2} c l\left(f_{E}\right)\right) \tilde{\subseteq} f_{E},
$$

and so $f_{E}$ is a $(1,2)^{*}$-fsp-closed set.

(ii). Since $f_{E}$ is a $(1,2)^{*}-f s r-o p e n ~ s e t, f_{E}=\tau_{1} \tau_{2} \operatorname{int}\left(\tau_{1} \tau_{2} c l\left(f_{E}\right)\right)$. Therefore, $f_{E} \tilde{\cap} \tau_{1} \tau_{2} c l\left(\tau_{1} \tau_{2} \operatorname{int}\left(f_{E}\right)\right) \tilde{\subseteq} f_{E}$. This implies, $\tau_{1} \tau_{2} \operatorname{cl}\left(\tau_{1} \tau_{2} \operatorname{int}\left(f_{E}\right)\right) \tilde{\subseteq} f_{E}$, and so $f_{E}$ is a $(1,2)^{*}$-fsp-closed set.

Theorem 4.6. Any $(1,2)^{*}$-fsb-closed set in fuzzy soft $\mathrm{f}_{\mathrm{E}}$ bitopological space $\left(\mathrm{X}, \mathrm{E}, \tau_{1}, \tau_{2}\right), \tau_{1} \tau_{2} \operatorname{int}\left(\mathrm{f}_{\mathrm{E}}\right)$ is a $(1,2)^{*}$ fsr-open set.

Proof. Let $f_{E}$ be a $(1,2)^{*}$-fsb-closed set, this implies $\tau_{1} \tau_{2} \operatorname{cl}\left(\tau_{1} \tau_{2} \operatorname{int}\left(f_{E}\right)\right) \tilde{\cap} \tau_{1} \tau_{2} \operatorname{int}\left(\tau_{1} \tau_{2} \operatorname{cl}\left(f_{E}\right)\right) \tilde{\subseteq} f_{E}$. Also, $\tau_{1} \tau_{2} \operatorname{int}\left(f_{E}\right) \tilde{\widetilde{c}} \tau_{1} \tau_{2} \operatorname{int}\left(\tau_{1} \tau_{2} \operatorname{cl}\left(\tau_{1} \tau_{2} \operatorname{int}\left(f_{E}\right)\right)\right) \tilde{\cap} \tau_{1} \tau_{2} \operatorname{int}\left(\tau_{1} \tau_{2} \operatorname{int}\left(\tau_{1} \tau_{2} c l\left(f_{E}\right)\right)\right) \tilde{\subseteq} \tau_{1} \tau_{2} \operatorname{int}\left(f_{E}\right)$.

Also, $\tau_{1} \tau_{2} \operatorname{int}\left(f_{E}\right) \tilde{\subseteq} \tau_{1} \tau_{2} \operatorname{int}\left(\tau_{1} \tau_{2} \operatorname{cl}\left(\tau_{1} \tau_{2} \operatorname{int}\left(f_{E}\right)\right)\right)$. Therefore, $\tau_{1} \tau_{2} \operatorname{int}\left(f_{E}\right)=\tau_{1} \tau_{2} \operatorname{int}\left(\tau_{1} \tau_{2} \operatorname{cl}\left(\tau_{1} \tau_{2} \operatorname{int}\left(f_{E}\right)\right)\right)$. So, $\tau_{1} \tau_{2}$ int $\left(f_{E}\right)$ is a $(1,2) *$-fsr-open set.

Theorem 4.7. Let $\mathrm{f}_{\mathrm{E}}$ be a $(1,2)^{*}$-fsb-closed set in fuzzy soft bitopological space $\left(\mathrm{X}, \mathrm{E}, \tau_{1}, \tau_{2}\right)$ if and only if $\mathrm{f}_{\mathrm{E}}$ is the intersection of $(1,2)^{*}$-fss-closed set and $(1,2)^{*}$-fsp-closed set.

Proof. Follows from the Definition 4.1.

\section{5. $(1,2)^{*}$-fuzzy soft b-interior and $(1,2)^{*}$-fuzzy soft b-closure}

Definition 5.1. Let $\left(X, E, \tau_{1}, \tau_{2}\right)$ be a fuzzy soft bitopological space and $f_{E} \widetilde{E} \widetilde{(X, E)}$.

(i) $(1,2)^{*}$-fuzzy soft b-closure (briefly $\left.(1,2)^{*}-f \operatorname{sbcl}\left(f_{E}\right)\right)$ of a set $f_{E}$ in $\left(X, E, \tau_{1}, \tau_{2}\right)$ defined by $(1,2)^{*}$ $f \operatorname{sbcl}\left(f_{E}\right)=\tilde{\cap}\left\{g_{E} \tilde{\simeq} f_{E}: g_{E}\right.$ is a $(1,2)^{*}$-fuzzy soft b-closed set in $\left.\left(X, E, \tau_{1}, \tau_{2}\right)\right\}$.

(ii) $(1,2)^{*}$-fuzzy soft b-interior (briefly $\left.(1,2)^{*}-f \operatorname{sbint}\left(f_{E}\right)\right)$ of a set $f_{E}$ in $\left(X, E, \tau_{1}, \tau_{2}\right)$ defined by $(1,2)^{*}$ $f \operatorname{sbint}\left(f_{E}\right)=\tilde{U}\left\{g_{E} \tilde{\subseteq} f_{E}: g_{E}\right.$ is a $(1,2)^{*}$-fuzzy soft b-open set in $\left.\left(X, E, \tau_{1}, \tau_{2}\right)\right\}$.

(iii) $(1,2)^{*}-f \operatorname{sbcl}\left(f_{E}\right)$ is the smallest $(1,2)^{*}$-fuzzy soft $b$-closed set in $\left(X, E, \tau_{1}, \tau_{2}\right)$ which contains $f_{E}$ and $(1,2)^{*}-f \operatorname{sbcl}\left(f_{E}\right)$ is the largest $(1,2)^{*}$-fuzzy soft $b$-closed set in $\left(X, E, \tau_{1}, \tau_{2}\right)$ which is contained in $f_{E}$.

The following lemma is used in the sequal.

Lemma 5.2. Let $\left(\mathrm{X}, \mathrm{E}, \tau_{1}, \tau_{2}\right)$ be a fuzzy soft bitopological space and $\mathrm{f}_{\mathrm{E}} \widetilde{\in} \widetilde{(\mathrm{X}, \mathrm{E})}$. Then

(i) $(1,2)^{*}-\operatorname{fscl}\left(f_{E}\right)=f_{E} \tilde{\cup} \tau_{1} \tau_{2} \operatorname{int}\left(\tau_{1} \tau_{2} \operatorname{cl}\left(f_{E}\right)\right)$;

(ii) $(1,2)^{*}-f \operatorname{ssint}\left(f_{E}\right)=f_{E} \tilde{\cap} \tau_{1} \tau_{2} c l\left(\tau_{1} \tau_{2} \operatorname{int}\left(f_{E}\right)\right)$;

(iii) $(1,2)^{*}-\operatorname{fscl}\left(f_{E}\right)=f_{E} \cup \tau_{1} \tau_{2} \operatorname{cl}\left(\tau_{1} \tau_{2} \operatorname{int}\left(f_{E}\right)\right)$;

(iv) $(1,2)^{*}-f \operatorname{spint}\left(f_{E}\right)=f_{E} \tilde{\cap} \tau_{1} \tau_{2} \operatorname{int}\left(\tau_{1} \tau_{2} \operatorname{cl}\left(f_{E}\right)\right)$. 
Theorem 5.3. Let $\left(\mathrm{X}, \mathrm{E}, \tau_{1}, \tau_{2}\right)$ be a fuzzy soft bitopological space and $\mathrm{f}_{\mathrm{E}} \tilde{\mathrm{E}} \widetilde{(\mathrm{X}, \mathrm{E})}$. Then

(i) $(1,2) *-f \operatorname{sbcl}\left(f_{E}\right)=f_{E} \tilde{U}\left[\tau_{1} \tau_{2} \operatorname{int}\left(\tau_{1} \tau_{2} c l\left(f_{E}\right)\right) \tilde{\cap} \tau_{1} \tau_{2} c l\left(\tau_{1} \tau_{2} \operatorname{int}\left(f_{E}\right)\right)\right]$;

(ii) $(1,2)^{*}-f \operatorname{sbint}\left(f_{E}\right)=f_{E} \tilde{\cap}\left[\tau_{1} \tau_{2} \operatorname{int}\left(\tau_{1} \tau_{2} \operatorname{cl}\left(f_{E}\right)\right) \tilde{U} \tau_{1} \tau_{2} c l\left(\tau_{1} \tau_{2} \operatorname{int}\left(f_{E}\right)\right)\right]$.

Proof. It is obvious.

Theorem 5.4. Let $\left(\mathrm{X}, \mathrm{E}, \tau_{1}, \tau_{2}\right)$ be a fuzzy soft bitopological space and $\mathrm{f}_{\mathrm{E}}, \mathrm{g}_{\mathrm{E}} \tilde{\in} \widetilde{(\mathrm{X}, \mathrm{E})}$. Then

(i) $(1,2)^{*}-f \operatorname{sbcl}\left(f_{E} \tilde{\cup} g_{E}\right) \simeq(1,2)^{*}-f \operatorname{sbcl}\left(f_{E}\right) \tilde{\cup}(1,2)^{*}-f \operatorname{sbcl}\left(g_{E}\right)$;

(ii) $(1,2)^{*}-f \operatorname{sbcl}\left(f_{E} \tilde{\cup} g_{E}\right) \tilde{\subseteq}(1,2)^{*}-f \operatorname{sbcl}\left(f_{E}\right) \tilde{\cap}(1,2)^{*}-f \operatorname{sbcl}\left(g_{E}\right)$;

(iii) $(1,2)^{*}-f \operatorname{sbint}\left(f_{E} \tilde{\cup} g_{E}\right) \tilde{\supseteq}(1,2)^{*}-f \operatorname{sbint}\left(f_{E}\right) \tilde{\cup}(1,2)^{*}-f \operatorname{sbint}\left(g_{E}\right)$;

(iv) $(1,2)^{*}-f \operatorname{sbint}\left(f_{E} \tilde{\cup} g_{E}\right) \tilde{\subseteq}(1,2)^{*}-f \operatorname{sbint}\left(f_{E}\right) \tilde{\cap}(1,2)^{*}-f \operatorname{sbint}\left(g_{E}\right)$.

Proof.

(i). Since $f_{E} \tilde{\subseteq} f_{E} \tilde{\cup} g_{E}$ or $g_{E} \tilde{\subseteq} f_{E} \tilde{\cup} g_{E}$. This implies, $(1,2)^{*}-f \operatorname{sbcl}\left(f_{E}\right) \tilde{\subseteq}(1,2)^{*}-f \operatorname{sbcl}\left(f_{E} \tilde{\cup} g_{E}\right)$ or $(1,2)^{*}-f \operatorname{sbcl}\left(g_{E}\right)$ $\tilde{\subseteq}(1,2)^{*}-f \operatorname{sbcl}\left(f_{E} \tilde{\cup} g_{E}\right)$. Thus, $(1,2)^{*}-f \operatorname{sbcl}\left(f_{E} \tilde{\cup} g_{E}\right) \tilde{\simeq}(1,2)^{*}-f \operatorname{sbcl}\left(f_{E}\right) \tilde{\cup}(1,2)^{*}-f \operatorname{sbcl}\left(g_{E}\right)$.

(ii). Similar to that of (i).

(iii). Since $f_{E} \tilde{\subseteq} f_{E} \tilde{\cup} g_{E}$ or $g_{E} \tilde{\subseteq} f_{E} \tilde{\cup} g_{E}$. This implies, $(1,2)^{*}-f \operatorname{sbint}\left(f_{E}\right) \tilde{\subseteq}(1,2)^{*}-f \operatorname{sbint}\left(f_{E} \tilde{\cup} g_{E}\right)$ or $(1,2)^{*}-f \operatorname{sbint}\left(g_{E}\right) \tilde{\subseteq}(1,2)^{*}-f \operatorname{sbint}\left(f_{E} \tilde{\cup} g_{E}\right)$. Thus, $(1,2)^{*}-f \operatorname{sbint}\left(f_{E} \tilde{\cup} g_{E}\right) \tilde{\Omega}(1,2)^{*}-f \operatorname{sbint}\left(f_{E}\right) \tilde{\cup}(1,2)^{*}-f \operatorname{sbint}\left(g_{E}\right)$. (iv). Similar to that of (iii).

Theorem 5.5. Let $\left(\mathrm{X}, \mathrm{E}, \tau_{1}, \tau_{2}\right)$ be a fuzzy soft bitopological space and $\mathrm{f}_{\mathrm{E}} \tilde{\in} \widetilde{(\mathrm{X}, \mathrm{E})}$. Then

(i) $\left[(1,2)^{*}-f \operatorname{sbcl}\left(f_{E}\right)\right]^{c}=\tilde{1}_{E} \backslash(1,2)^{*}-f \operatorname{sbint}\left(f_{E}\right)$;

(ii) $\left[(1,2)^{*}-f \operatorname{sbint}\left(f_{E}\right)\right]^{c}=\tilde{1}_{E} \backslash(1,2)^{*}-f \operatorname{sbcl}\left(f_{E}\right)$.

Proof.

(i). Let $f_{E} \tilde{\in} \widetilde{(X, E)} \cdot(1,2)^{*}-f \operatorname{sbcl}\left(f_{E}\right)=\tilde{n}\left\{g_{E} \supseteq f_{E}: g_{E}\right.$ is a $(1,2)^{*}$-fuzzy soft b-closed set in $\left.\left(X, E, \tau_{1}, \tau_{2}\right)\right\}$. This implies

$$
\begin{aligned}
{\left[(1,2)^{*}-f \operatorname{sbcl}\left(f_{E}\right)\right]^{c} } & =\left[\tilde{\cap}\left\{g_{E} \supseteq f_{E}: g_{E} \text { is a }(1,2)^{*} \text {-fuzzy soft b-closed set in }\left(X, E, \tau_{1}, \tau_{2}\right)\right\}\right]^{c} \\
& =\left[\tilde{U}\left\{g_{E}^{c} \supseteq f_{E}^{c}: g_{E}^{c} \text { is a }(1,2)^{*}-\text { fuzzy soft b-open set in }\left(X, E, \tau_{1}, \tau_{2}\right)\right\}\right]^{c} \\
& =\left[(1,2)^{*}-f \operatorname{sbcl}\left(f_{E}\right)\right]^{c}=\tilde{1}_{E} \backslash(1,2)^{*}-f \operatorname{sbint}\left(f_{E}\right) .
\end{aligned}
$$

(ii). We get the result by applying fuzzy soft interior in (i).

Theorem 5.6. Let $\left(\mathrm{X}, \mathrm{E}, \tau_{1}, \tau_{2}\right)$ be a fuzzy soft bitopological space and $\mathrm{f}_{\mathrm{E}} \widetilde{\in(X, E)}$. Then

(i) $(1,2)^{*}-f \operatorname{sbcl}\left(f_{E}\right) \tilde{\subseteq}(1,2)^{*}-f \operatorname{sscl}\left(f_{E}\right) \tilde{\cap}(1,2)^{*}-f \operatorname{spcl}\left(f_{E}\right)$;

(ii) $(1,2)^{*}-f \operatorname{sbint}\left(f_{E}\right) \tilde{\supseteq}(1,2)^{*}-f \operatorname{ssint}\left(f_{E}\right) \tilde{\cup}(1,2)^{*}-f \operatorname{spint}\left(f_{E}\right)$.

Proof.

(i).

$$
\begin{aligned}
(1,2)^{*}-f \operatorname{sscl}\left(f_{E}\right) \tilde{\cap}(1,2)^{*}-f \operatorname{spcl}\left(f_{E}\right) \tilde{\supseteq}\left[\left(f_{E} \tilde{\cup} \tau_{1} \tau_{2} \operatorname{int}\left(\tau_{1} \tau_{2} \operatorname{cl}\left(f_{E}\right)\right)\right) \tilde{\cap}\left(f_{E} \tilde{\cup} \tau_{1} \tau_{2} \operatorname{cl}\left(\tau_{1} \tau_{2} \operatorname{int}\left(f_{E}\right)\right)\right)\right] \\
=f_{E} \tilde{\cup}\left[\tau_{1} \tau_{2} \operatorname{int}\left(\tau_{1} \tau_{2} \operatorname{cl}\left(f_{E}\right)\right) \tilde{\cap} \tau_{1} \tau_{2} \operatorname{cl}\left(\tau_{1} \tau_{2} \operatorname{int}\left(f_{E}\right)\right)\right]=(1,2)^{*}-\operatorname{fsbcl}\left(f_{E}\right) .
\end{aligned}
$$

(ii).

$$
\begin{array}{r}
(1,2)^{*}-f \operatorname{sint}\left(f_{E}\right) \tilde{\cup}(1,2)^{*}-f \operatorname{spint}\left(f_{E}\right) \tilde{\subseteq}\left[\left(f_{E} \tilde{\cap} \tau_{1} \tau_{2} \operatorname{cl}\left(\tau_{1} \tau_{2} \operatorname{int}\left(f_{E}\right)\right)\right) \tilde{\cup}\left(f_{E} \tilde{\cap} \tau_{1} \tau_{2} \operatorname{int}\left(\tau_{1} \tau_{2} c l\left(f_{E}\right)\right)\right)\right] \\
=f_{E} \tilde{\cap}\left[\tau_{1} \tau_{2} \operatorname{cl}\left(\tau_{1} \tau_{2} \operatorname{int}\left(f_{E}\right)\right) \tilde{\cup} \tau_{1} \tau_{2} \operatorname{int}\left(\tau_{1} \tau_{2} \operatorname{cl}\left(f_{E}\right)\right)\right]=(1,2)^{*}-f \operatorname{sint}\left(f_{E}\right) .
\end{array}
$$


Theorem 5.7. Let $\left(\mathrm{X}, \mathrm{E}, \tau_{1}, \tau_{2}\right)$ be a fuzzy soft bitopological space and $\mathrm{f}_{\mathrm{E}} \tilde{\mathrm{E}} \widetilde{(\mathrm{X}, \mathrm{E})}$. Then

(i) $f_{E}$ is $(1,2)^{*}$-fsb-closed if and only if $f_{E}=(1,2)^{*}-f \operatorname{sbcl}\left(f_{E}\right)$;

(ii) $f_{\mathrm{E}}$ is $(1,2)^{*}-\mathrm{fsb}$-open if and only if $\mathrm{f}_{\mathrm{E}}=(1,2)^{*}-\mathrm{f} \operatorname{sbint}\left(\mathrm{f}_{\mathrm{E}}\right)$.

Proof. Suppose $f_{E}=(1,2)^{*}-f \operatorname{sbcl}\left(f_{E}\right)=\tilde{n}\left\{g_{E} \supseteq f_{E}: g_{E}\right.$ is a $(1,2)^{*}-f s b$-closed set in $\left.\left(X, E, \tau_{1}, \tau_{2}\right)\right\}$. Therefore, $f_{E}$ is $(1,2)^{*}$-fsb-closed. Conversely, suppose $f_{E}$ is $(1,2)^{*}$-fsb-closed in $\left(X, E, \tau_{1}, \tau_{2}\right)$. If we take $f_{E} \tilde{\subseteq} f_{E}$ and $f_{E}$ is $(1,2)^{*}-f s b$-closed. Therefore, $f_{E} \tilde{\in} \tilde{\cap}\left\{g_{E} \supseteq f_{E}: g_{E}\right.$ is a $(1,2)^{*}-f$ sb-closed set in $\left.\left(X, E, \tau_{1}, \tau_{2}\right)\right\}$. $f_{E} \tilde{\subseteq} g_{E}$ implies, $f_{E}=\tilde{\cap}\left\{g_{E} \supseteq f_{E}: g_{E}\right.$ is a $(1,2)^{*}-f s b$-closed set in $\left.\left(X, E, \tau_{1}, \tau_{2}\right)\right\}=(1,2)^{*}-f \operatorname{sbcl}\left(f_{E}\right)$.

\section{Conclusion}

In this paper, fuzzy soft b-open sets in fuzzy soft bitopological space are defined and developed. We then presented their properties and compared their relations with some other types of fuzzy soft sets. In future, using these sets; various classes of mappings on fuzzy soft bitopological space can be studied.

We hope that this paper is just a beginning of a new structure and there are scopes for a researcher in this field.

\section{Acknowledgment}

The author is very grateful to the editor and the reviewers for their valuable suggestions.

\section{References}

[1] A. A'ikgöz, N. A. Taş, Some new mixed soft sets, Math. Sci. Appl. E-Notes, 2 (2014), 105-118. 1

[2] A. A'ikgöz, N. A. Taş, T. Noiri, A decomposition of some types of mixed soft continuity in soft topological spaces, Filomat, 30 (2016), 379-385. 1

[3] T. Beaulaa, C. Gunaseelib, On fuzzy soft metric spaces, Malaya J. Mat., 44 (2014), 197-202. 2.12

[4] D. G. Chen, E. C. C. Tsang, D. S. Yeung, X. Z. Wang, The parameterization reduction of soft sets and its applications, Comput. Math. Appl., 49 (2005), 757-763. 1

[5] C. C. Chou, J. M. Yih, J. F. Ding, T. C. Han, Y. H. Lin, L. J. Liu, W. K. Hsu, Application of a fuzzy EOQ model to the stock management in the manufacture system, Key Eng. Mat., 499 (2012), 361-365. 1

[6] C. Eksin, M. Güzelkaya, E. Yesil, I. Eksin, Fuzzy logic approach to mimic decision making behaviour of humans in stock management game, Proceedings of System Dynamics Conference, 2008 (2008), 20 pages. 1

[7] B. M. Ittanagi, Soft Bitopological Spaces, Int. J. Comput. Appl., 107 (2014), 1-4. 1

[8] A. Kandil, O. A. E. Tantawy, S. A. El-Sheikh, S. A. Hazza, Pairwise open (closed) soft sets in soft bitopological spaces, Ann. Fuzzy Math. Inform., 11 (2016), 571-588. 1

[9] A. Kandil, O. A. E. Tantawy, S. A. El-Sheikh, S. A. Hazza, Pairwise soft separation axioms in soft bitopological spaces, Ann. Fuzzy Math. Inform., 13 (2017), 563-577. 1

[10] J. C. Kelly, Bitopological spaces, Proc. London Math. Soc. (3), 13 (1963), 71-81. 1

[11] J. Mahanta, P. K. Das, Results on fuzzy soft topological spaces, arXiv, 2012 (2012), 11 pages. 2.10, 2.11

[12] P. K. Maji, R. Biswas, A. R. Roy, Fuzzy soft sets, J. Fuzzy Math., 9 (2001), 589-602. 2.4

[13] P. K. Maji, R. Biswas, A. R. Roy, Soft set theory, Comput. Math. Appl., 45 (2003), 555-562. 1, 2.5

[14] D. Molodtsov, Soft set theory-first results, Comput. Math. Appl., 37 (1999), 19-31. 1, 2.3

[15] P. Mukherjee1, C. Park, On fuzzy soft Bitopological spaces, Math. Comput. Sci. J., 10 (2015), 1-8. 1, 2.14, 2.15, 2.16, $2.17,2.18,2.19,2.20,2.21,2.22,2.23,2.24,2.25$

[16] N. Y. Özgür, N. Taş, A note on "application of fuzzy soft sets to investment decision making problem", J. New Theory, 7 (2015), 1-10. 1

[17] C. W. Patty, Bitopological spaces, Duke Math. J., 34 (1967), 387-391. 1

[18] I. L. Reilly, On bitopological separation properties, Nanta Math., 5 (1972), 14-25. 1

[19] N. Revathi, K. Bageerathi, On Soft B-Open Sets In Soft Bitopological Space, Int. J. Appl. Res., 11 (2015), 615-623. 1

[20] S. Roy, T. K. Samanta, A note on fuzzy soft topological spaces, Ann. Fuzzy Math. Inform., 3 (2012), 305-311. 2.6, 2.7, $2.8,2.9,2.13$

[21] A. F. Sayed, On characterizations of some types of fuzzy soft Sets in fuzzy soft bitopological spaces, J. Adv. Math. Comput. Sci., 24 (2017), 1-12. 1, 2.26

[22] A. F. Sayed, Some separation axioms in fuzzy soft bitopological spaces, J. Math. Comput. Sci., 8 (2018), $28-45.1$

[23] N. A. Taş, A. A'1kgöz, Some mixed soft operations and extremally soft disconnectedness via two soft topologies, Appl. Math., 5 (2014), 490-500. 1 
[24] S. Yüksel, T. Dizman, G. Yildizdan, U. Sert, Application of soft sets to diagnose the prostate cancer risk, J. Inequal. Appl., 2013 (2013), 11 pages. 1

[25] L. A. Zadeh, Fuzzy sets, Inf. Control, 8 (1965), 338-353. 1, 2.1, 2, 2.2 